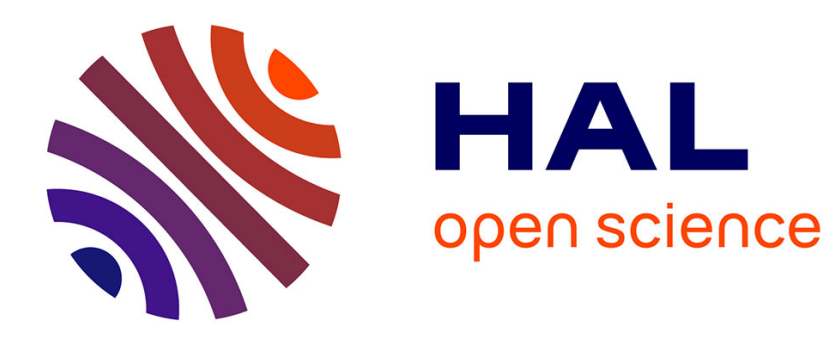

\title{
Lovely pairs of models: the non first order case
}

Itaï Ben Yaacov

\section{To cite this version:}

Itaï Ben Yaacov. Lovely pairs of models: the non first order case. The Journal of Symbolic Logic, 2004, 69 (3), pp.641-662. hal-00357704

\section{HAL Id: hal-00357704 https://hal.science/hal-00357704}

Submitted on 1 Feb 2009

HAL is a multi-disciplinary open access archive for the deposit and dissemination of scientific research documents, whether they are published or not. The documents may come from teaching and research institutions in France or abroad, or from public or private research centers.
L'archive ouverte pluridisciplinaire HAL, est destinée au dépôt et à la diffusion de documents scientifiques de niveau recherche, publiés ou non, émanant des établissements d'enseignement et de recherche français ou étrangers, des laboratoires publics ou privés. 


\title{
LOVELY PAIRS OF MODELS: THE NON FIRST ORDER CASE
}

\author{
ITAY BEN-YAACOV
}

\begin{abstract}
We prove that for every simple theory $T$ (or even simple thick compact abstract theory) there is a (unique) compact abstract theory $T^{\mathfrak{P}}$ whose saturated models are the lovely pairs of $T$. Independence-theoretic results that were proved in [BPV03] when $T^{\mathfrak{P}}$ is a first order theory are proved for the general case: in particular $T^{\mathfrak{P}}$ is simple and we characterise independence.
\end{abstract}

\section{INTRODUCTION}

Lovely pairs of models of a simple first order theory were defined in [BPV03]. Under an additional assumption, namely that the equivalent conditions of Fact 0.2 below hold, it is shown that lovely pairs provide an elegant means for the study of independencerelated phenomena in such a theory. This generalises a similar treatment of stable theories through the study of beautiful pairs in [Poi83].

A lovely pair of models of $T$ is given by $(M, P)$ where $M \vDash T$ and $P$ is a new unitary predicate defining an elementary sub-structure with quite a few additional properties (see Definition 2.1 below). The following is proved in [BPV03] (the analogue for beautiful pairs of models of a stable theory is proved in [Poi83]):

Fact 0.1. Let $T$ be a complete simple first order theory. Then all lovely pairs of $T$ have the same first order theory $T^{P}$ in the language $\mathcal{L} \cup\{P\}$.

This does not mean, however, that the complete first order theory $T^{P}$ is meaningful. For example, in order to use $T^{P}$ for the study of lovely pairs we would like saturated models of $T^{P}$ to be ones. In fact, it is proved that:

Fact 0.2. The following conditions are equivalent (for a first order simple theory $T$ ):

(i) The $|T|^{+}$-saturated models of $T^{P}$ are precisely the lovely pairs.

(ii) There is a $|T|^{+}$-saturated model of $T^{P}$ which is a lovely pair.

(iii) The notion of elementary extension of models of $T^{P}$ coincides with that of a free extension (Definition 1.8).

(iv) Every model of $T^{P}$ embeds elementarily in a lovely pair.

Date: February 1, 2009.

2000 Mathematics Subject Classification. 03C45,03C95.

Key words and phrases. simple theories - lovely pairs.

This is the result of research conducted in the University of Illinois at Urbana-Champaign, as a part of CNRS-UIUC collaboration. The author would like to thank Anand Pillay and Evgueni Vassiliev for hospitality and fruitful discussions.

At the time of the writing of this paper, the author was a graduate student with the Équipe de Logique Mathématique of Université Paris VII. 
If this holds (the "good" case), then $T^{P}$ is simple as well, and provides an elegant means for the study of certain independence-related properties of $T$ itself, as mentioned above. If this fails (the "bad" case), then the first order theory $T^{P}$ is pretty much useless. This was noticed by Poizat in the stable case, where things go well if and only if $T$ does not have the finite cover property; the analogous criterion for simple theories can be argued to be the correct analogue of non-f.c.p. in simple theories.

The goal of the present article is to show that in the proper context, one can do in the "bad" case just the same things as in the "good" one. By the previous discussion it should be clear that this cannot be done in first order model theory, and we need to look for a more general framework. Such a framework, that of compact abstract theories, or cats, is exposed in Ben03a. Simplicity theory is developed for cats in Ben03b, but has a few setbacks with respect to first order simplicity. In Bena we define the notion of a thick cat (which is still much more general than a first order theory), and prove all basic properties of simplicity theory in this framework.

Here we prove that if $T$ is a thick simple cat (so in particular, if $T$ is a simple first order theory), then there exists a unique cat $T^{\mathfrak{P}}$, whose saturated models are precisely the lovely pairs of $T . T^{\mathfrak{P}}$ is also thick and simple and has a language of the same cardinality as $T$. In addition, there is a description (a notion close to interpretation, defined in [Bena]) of $T^{\mathfrak{P}}$ in $T$, which gives us an elegant characterisation of independence in $T^{\mathfrak{P}}$. We also prove that if $T$ is Hausdorff, semi-Hausdorff, supersimple, stable, stable and Robinson, or one-based, then so is $T^{\mathfrak{P}}$.

It follows from Proposition 2.3 below that $T^{\mathfrak{P}}$ is (equivalent to) a first order theory if and only if $T$ is and the equivalent conditions of Fact 0.2 hold. Thus, for a first order theory $T$, the "good" and "bad" cases are simply the first order case and the non-first-order one, respectively, of which the former was studied in [BPV03]. In the present paper, however, such considerations as whether $T^{\mathfrak{P}}$ is first order or not are hardly of any importance.

The fundamental tool is the construction of a cat from a compact abstract elementary category, as described in Ben03a. This tool allows us in certain cases to fix the notion of elementary extension as we like: since we know that things go well if and only if the elementary extensions are the free extensions, we turn things around and try to construct a cat where free extensions play the role of elementary ones. As it turns out, this is indeed one of the cases where this technique works, and the only assumption on the original theory we actually use is that it is a thick simple cat.

We can think of several reasons why this may be an interesting thing to do: First, this gives a nice and rather comprehensive set of examples of the basic tools used in the framework of cats, and in particular of simplicity theory. Second, this is an additional example supporting our thesis that simplicity in thick cats lacks nothing in comparison with simplicity in first order theories (alas, this is not true for simplicity in arbitrary cats). Third, and maybe most important, thick simple cats are (or at least, seem to be) the correct framework for the treatment of lovely pairs, and therefore results proved in this context should be the most general.

Moreover, this framework allows us to state and prove results that are either unnatural or altogether meaningless in the first order case. Even when proving something that makes perfect sense in a first order theory, we may use for its proof tools that would be unnatural in the treatment of a first order theory, and this may eventually yield 
a simpler or more elegant proof. In fact, some results appearing in BPV03] (notably the preservation of one-basedness) were originally proved quite easily in this context, and it took a bit of effort to find first order counterparts for the "feline" proofs.

Let us give a few reminders concerning cats. Most of this comes from Ben03a.

Definition 0.3. Let $\mathcal{L}$ be a first order language, and fix a positive fragment of $\mathcal{L}$, i.e., a subset $\Delta \subseteq \mathcal{L}$ which is closed for positive boolean combinations (actually, $\mathcal{L}$ is completely unimportant, all we want is $\Delta$ ). A formula, unless otherwise qualified, is always a member of $\Delta$, and similarly for partial types.

A universal domain (with respect to $\Delta$ ) is a structure $U$ satisfying:

(i) Strong homogeneity: If $A, B \subseteq U$ are small and $f: A \rightarrow B$ is a $\Delta$ homomorphism (i.e., for every $\varphi \in \Delta$ and $a \in A, U \vDash \varphi(a) \Longrightarrow U \vDash \varphi(f(a))$ ), then $f$ extends to an automorphism of $U$ (so in particular, $f$ is a $\Delta$ isomorphism of $A$ and $B$ ).

(ii) Compactness: Every small partial $\Delta$-type over $U$ which is finitely realised in $U$ is realised in $U$.

Although this is not required by the definition, we will also assume that every existential formula, i.e., formula of the form $\exists y \varphi(x, y)$ where $\varphi \in \Delta$, is equivalent in $U$ to a partial $\Delta$-type. (If not, we can always close $\Delta$ under existential quantification without harming either compactness or homogeneity; this is just usually unnecessary.)

Saturated and strongly homogeneous models of first order theories are one example of universal domain (with $\Delta=\mathcal{L}$ ). Another easy example which we will refer to later on is that of Hilbert spaces:

Example 0.4. Let $H$ be the unit ball of a very large Hilbert space. Let $\Delta$ be the set of all formulas of the form $s \leq\left\|\sum_{i<n} \lambda_{i} x_{i}\right\| \leq r$ (closed under positive boolean combinations). Then $H$ is a universal domain w.r.t. $\Delta$.

The negative universal theory of a universal domain

$$
\operatorname{Th}_{\Pi}(U)=\{\forall \bar{x} \neg \varphi: \varphi(\bar{x}) \in \Delta, U \vDash \forall \bar{x} \neg \varphi(\bar{x})\}
$$

has the property that the category of subsets of its e.c. models has the amalgamation property (there is a little twist here, since the notion of e.c. models is defined with respect to $\Delta$-homomorphisms). A negative universal theory having this property is called a positive Robinson theory. Conversely, if $T$ is a positive Robinson theory, and in addition is complete (i.e., the category of its e.c. models has the joint embedding property), then $T=\operatorname{Th}_{\Pi}(U)$ for some universal domain $U$; otherwise, every completion of $T$ has a universal domain. Thus the giving of a universal domain is essentially the same as the giving of a complete positive Robinson theory. Henceforth, a theory means a positive Robinson theory, unless explicitly stated otherwise.

To a universal domain $U$, or to a theory $T$, we associate type-spaces: for every set of indices $I$ we define $\mathrm{S}_{I}(T)$ as the set of all maximal types in $\alpha$ variables which are consistent with $T$. If $U$ is a universal domain for $T$ then this is the same as $U^{I} / \operatorname{Aut}(U)$, by homogeneity. We put a compact and $T_{1}$ topology on $\mathrm{S}_{I}(T)$ by taking the closed sets to be those defined by partial types. If $\mathrm{S}_{n}(T)$ is Hausdorff for every $n<\omega$ then $\mathrm{S}_{I}(T)$ is Hausdorff for every set $I$, and we say that $T$ is Hausdorff. One consequence of being 
Hausdorff is that the property of two tuples to have the same type is a type-definable property. If only the latter holds, we say that $T$ is semi-Hausdorff. An even weaker property is thickness, defined in [Bena], which says that indiscernibility of sequences is type-definable.

We render the mapping $I \mapsto \mathrm{S}_{I}(T)$ a contravariant functor in the obvious manner: if $f: I \rightarrow J$ is any mapping, then $f^{*}: \operatorname{tp}\left(a_{j}: j \in J\right) \mapsto \operatorname{tp}\left(a_{f(i)}: i \in I\right)$ defines a continuous mapping $f^{*}: \mathrm{S}_{J}(T) \rightarrow \mathrm{S}_{I}(T)$. We call this the type-space functor of $T$, denoted $\mathrm{S}(T)$. Conversely, up to a change of language, we can reconstruct the positive Robinson theory $T$ from $\mathrm{S}(T)$ (see [Ben03a, Theorem 2.23]).

Finally, in Ben03a, Section 2.3] we characterise when a class of structures equipped with a notion of embedding has a universal domain which is also a universal domain for a positive Robinson theory. First, we represent such a class with concrete category $\mathcal{M}$ all of whose morphisms are injective (the embeddings); we call it an elementary category with amalgamation if it satisfies some additional properties: Tarski-Vaught, elementary chain and amalgamation (see [Ben03a, Definition 2.27]). In particular, amalgamation gives us a reasonably good notion of type: if $M$ and $N$ are models (i.e., objects of $\mathcal{M}$ ) and $\bar{a} \in M$ and $\bar{b} \in N$ are tuples of the same length, then they have the same type if and only if we can embed $M$ and $N$ in a third model $P$ such that the images of $\bar{a}$ and $\bar{b}$ in $P$ coincide. This defines a contravariant functor $\mathrm{S}(\mathcal{M})$ from sets to sets as above. Using this notion of types we obtain some rudimentary semantics that allow us to state the three last requirements (see [Ben03a, Definition 2.32]): that the collection of types is not a proper class; that types of infinite tuples are determined by the types of finite sub-tuples; and most importantly, that we can put compact and $T_{1}$ topologies on each $\mathrm{S}_{I}(\mathcal{M})$ such that its morphisms are closed continuous mappings. This last requirement is equivalent to saying that there is a language $\mathcal{L}$, a positive fragment $\Delta \subseteq \mathcal{L}$, and a way to render every object of $\mathcal{M}$ an $\mathcal{L}$-structure, such that:

(i) The satisfaction of a $\Delta$-formula by a tuple in $M \in \mathcal{M}$ is determine by the type of the tuple.

(ii) If $\Sigma$ is a set of $\Delta$-formulas, possibly in infinitely many variables, and $\Sigma$ is finitely realised in $\mathcal{M}$, then it is realised in $\mathcal{M}$.

If all these requirements hold, then there exists a positive Robinson theory $T$ (in fact $T=\mathrm{Th}_{\Pi}(\mathcal{M})$ ) satisfying $\mathrm{S}(T) \cong \mathrm{S}(\mathcal{M})$, and e.c. models of $T$ embed in models of $\mathcal{M}$ and vice versa. Also, $T$ is complete if and only if $\mathcal{M}$ has the joint embedding property, and in this case a universal domain for $T$ is a universal domain for $\mathcal{M}$ in some reasonable sense.

Since we have three equivalent presentation (a positive Robinson theory, a compact type-space functor and a compact elementary category) of the same concept, we prefer to refer to this concept with a generic name: compact abstract theory, or cat. The third presentation of cats is the main tool we use in the first section. In the fourth section we concentrate on the second approach and study the relations between the type-space functors of our theory $T$ and of the theory of its pairs $T^{\mathfrak{P}}$.

As for simplicity and independence, the thumb rule is that everything that's true in a simple first order theory (by which we mean the main results of [Kim98, KP97, HKP00]) is true in a simple thick cat. Part of this is shown for arbitrary simple cats in Ben03b 
and the rest (in particular the extension axiom) is shown in Bena under the hypothesis of thickness.

We consider the distinction between the "real" sorts and the hyperimaginary sorts immaterial: an element is usually a real one, but as we may adjoin any hyperimaginary sort to the original theory it may in fact be in any such sort. We use lowercase letters to denote elements and (possibly infinite) tuples thereof, and uppercase letters to denote sets of such elements or tuples (of course, any set can be enumerated into a tuple, but sometimes it's convenient to make a conceptual distinction).

We recall that if $a$ is a tuple of elements, or even a hyperimaginary element, then $\operatorname{bdd}(a)$ (respectively $\operatorname{dcl}(a)$ ) is the collection of all hyperimaginary elements $b$ such that $\operatorname{tp}(b / a)$ has boundedly many realisations (respectively, a unique realisation). If $A \subseteq B$ then $A$ is boundedly closed in $B$ if $B \cap \operatorname{bdd}(A)=A$.

It is also a fact that if $c \in \operatorname{bdd}(b)$ then $a \downarrow_{b} c$ for every $a$, and $a \downarrow_{b} a$ if and only if $a \in \operatorname{bdd}(b)$.

\section{The CATEGORY OF T-PAIRS}

Convention 1.1. We fix a thick simple cat $T$.

We may consider it as a positive Robinson theory with respect to a positive fragment $\Delta$. By an elementary mapping we mean a $\Delta$-elementary one, that is a $\Delta$-homomorphism.

We do not assume that $T$ is complete. Therefore, instead of working inside a single universal domain for $T$, we work with the category of e.c. models of $T$ (or more precisely, of subsets thereof). The reader should keep in mind the existence of a partial elementary mapping between two e.c. models of $T$ implies that they are models of the same completion, so we could assume that $T$ is complete without much loss of generality.

We aim at the construction of $T^{\mathfrak{P}}$. Our starting point is the notions of pair and free extension/embedding:

Definition 1.2. $\quad$ (i) A pair is a couple $(A, P)$ where $A$ is a subset of some e.c. model of $T$, and $P$ is a unary predicate on $A$, such that $P(A)$ is boundedly closed in $A$ (i.e., $A \cap \operatorname{bdd}(P(A))=P(A))$. We allow ourselves to omit $P$ when no ambiguity may arise, convening that it is part of the structure on $A$.

(ii) A free embedding of pairs $f:(A, P) \rightarrow(B, P)$ is an elementary embedding $f$ : $A \rightarrow B$ such that $f(P(A)) \subseteq P(B)$ and $f(A) \downarrow_{f(P(A))} P(B)$. (Independence here is calculated in $B$, i.e., in any e.c. model or universal domain in which $B$ is embedded.)

(iii) The free category of pairs, $\mathfrak{P}$, is the category whose objects are pairs and whose morphisms are free embeddings.

Lemma 1.3. Assume that $f:(A, P) \rightarrow(B, P)$ is a free embedding. Then $P(f(A))=$ $f(P(A))=f(A) \cap P(B)$.

Proof. Clearly $f(P(A)) \subseteq P(B) \cap f(A)=P(f(A))$, whereby

$$
f(A) \underset{f(P(A))}{\downarrow} P(B) \Longrightarrow P(f(A)) \underset{f(P(A))}{\downarrow} P(f(A)) \Longrightarrow P(f(A)) \subseteq \operatorname{bdd}(f(P(A)))
$$


But then $P(f(A))$ is a subset of:

$$
\operatorname{bdd}(f(P(A))) \cap f(A)=f(\operatorname{bdd}(P(A)) \cap A)=f(P(A))
$$

And the claim ensues.

It follows that if the inclusion $\left(A, P_{A}\right) \subseteq\left(B, P_{B}\right)$ is a free embedding, then $P_{B}$ agrees with $P_{A}$ on $A$, and it is legitimate to use $P$ without further qualification.

Remark 1.4. One easily verifies that the identity is a free embedding, as well as the composition of any two free embeddings $f: A \rightarrow B$ and $g: B \rightarrow C: g(f(P(A))) \subseteq$ $g(P(B)) \subseteq P(C)$ and $f(A) \downarrow_{f(P(A))} P(B) \Longrightarrow g(f(A)) \downarrow_{g(f(P(A)))} g(P(B))$, so $g(B) \downarrow_{g(P(B))} P(C) \Longrightarrow g(f(A)) \downarrow_{g(f(P(A)))} P(C)$ by transitivity.

Since in a free embedding we have $f(A) \cap P(B)=f(P(A))$, we may usually assume that a free embedding is in fact an inclusion.

We aim to prove that $\mathfrak{P}$ is a compact elementary category, as defined in Ben03a.

Proposition 1.5. $\mathfrak{P}$ is an abstract elementary category with amalgamation ([Ben03a, Definition 2.27]).

Proof. Clearly, $\mathfrak{P}$ is a concrete category; we verify the properties:

Injectiveness: Every free embedding is injective.

Tarski-Vaught property: Assume that we have free inclusions $A \subseteq C$ and $B \subseteq C$, such that $A \subseteq B$, and we need to show that the inclusion $A \subseteq B$ is free as well. It is clearly elementary, $P(A) \subseteq P(B)$ and $A \downarrow_{P(A)} P(C) \Longrightarrow$ $A \downarrow_{P(A)} P(B)$.

Elementary chain property: Let $\left(A_{i}, P\right)$ be pairs for $i<\lambda, A_{i} \subseteq A_{j}$ freely for every $i \leq j<\lambda$, and set $(B, P)=\bigcup_{i<\lambda}\left(A_{i}, P\right)$. By the finite character of dividing $A_{i} \downarrow_{P\left(A_{i}\right)} P(B) \Longrightarrow \operatorname{bdd}\left(P(B) \cap A_{i}\right)=P\left(A_{i}\right)$ for every $i$, so $P(B)$ is boundedly closed in $B$, and $(B, P)$ is a pair. Clearly $A_{i} \subseteq B$ is a free extension for every $i$, and $(B, P)$ is clearly minimal as such.

Amalgamation: Assume $f: A \rightarrow B$ and $g: A \rightarrow C$ are free. We may embed $\left(A, P_{A}\right),\left(B, P_{B}\right)$ and $\left(C, P_{C}\right)$ in an appropriate universal domain of $T$ such that $f$ and $g$ be the identity maps and $B \downarrow_{A} C$. We know that both $P_{B}$ and $P_{C}$ coincide with $P_{A}$ on $A$, but we still do not know that they coincide on $B \cap C$, so let us keep the distinction for a while. Define $D=B \cup C$ and $P_{D}=P_{B} \cup P_{C}$. Then we have:

$$
B \underset{A}{\downarrow} C \Longrightarrow B \underset{A}{\downarrow} P_{C} \Longrightarrow B \underset{P_{A}}{\downarrow} P_{C} \Longrightarrow B \underset{P_{B}}{\downarrow} P_{D}
$$

And similarly $C \downarrow_{P_{C}} P_{D}$. Also, if $a \in D \cap \operatorname{bdd}\left(P_{D}\right)$ then either $a \in B$ or $a \in C$. In the former case:

$$
B \underset{P_{B}}{\downarrow} P_{D} \Longrightarrow a \underset{P_{B}}{\downarrow} a \Longrightarrow a \in \operatorname{bdd}\left(P_{B}\right) \Longrightarrow a \in P_{B}
$$

and in the latter $a \in P_{C}$, so in either case $a \in P_{D}$. This shows that $D \cap$ $\operatorname{bdd}\left(P_{D}\right)=P_{D}$, so $\left(D, P_{D}\right)$ is a pair, and the inclusions $B \subseteq D$ and $C \subseteq D$ are free. (It follows now by Lemma 1.3 that $P_{A}, P_{B}$ and $P_{C}$ are simply $P_{D}$ restricted to $A, B$ and $C$, respectively.) 
$\mathrm{QED}_{1.5}$

Therefore we have a notion of type: we recall that $\operatorname{tp}^{(A, P)}(a)=\operatorname{tp}^{(B, P)}(b)$ if there are free embeddings of $(A, P)$ and $(B, P)$ into some pair $(C, P)$ such that $a$ and $b$ have the same image, and $\mathrm{S}_{\alpha}(\mathfrak{P})$ is the set (or class, as far as we know at this point) of types of $\alpha$-tuples in $\mathfrak{P}$.

The next step is to understand types:

Definition 1.6. Let $(A, P)$ be a pair, and $a \in A$ a tuple.

(i) $a^{c}=\mathrm{Cb}(a / P(A))$ (calculated in $\left.T\right)$.

As $a^{c} \in \operatorname{bdd}(P(A)) \cap \operatorname{dcl}(a P(A)) \subseteq \operatorname{dcl}(A)$, this definition takes place entirely within $A$; and since the canonical base over $P$ is invariant under free extensions, we may write it rather as $a^{c}=\mathrm{Cb}(a / P)$ without concerning ourselves in which specific pair this is taken.

(ii) $\hat{a}=a, a^{c}$.

(iii) The Morley class $\operatorname{mcl}(a)$ is the set of pure types of Morley sequences (of length $\omega)$ in $\operatorname{tp}\left(a / a^{c}\right)$.

Lemma 1.7. If $(A, P)$ and $a \in A$ are as above, then $\operatorname{mcl}(a)$ is the set of types of Morley sequences in $\operatorname{tp}(a / P(A))$.

Proof. Easy.

$\mathrm{QED}_{1.7}$

Lemma 1.8. Let $(A, P)$ and $(B, P)$ be two pairs, and $a \in A, b \in B$ be two possibly infinite tuples. Then the following are equivalent:

(i) $\operatorname{tp}^{(A, P)}(a)=\operatorname{tp}^{(B, P)}(b)$ (in the sense of $\left.\mathfrak{P}\right)$.

(ii) $\mathrm{mcl}^{(A, P)}(a)=\mathrm{mcl}^{(B, P)}(b)$

(iii) $\mathrm{mcl}^{(A, P)}(a) \cap \mathrm{mcl}^{(B, P)}(b) \neq \varnothing$

(iv) $\operatorname{tp}^{T}\left(\hat{a}^{(A, P)}\right)=\operatorname{tp}^{T}\left(\hat{b}^{(B, P)}\right)$.

Proof. $\quad$ (i) $\Longrightarrow$ (ii). mcl is invariant under free extensions.

(ii) $\Longrightarrow$ (iii). Morley sequences exist.

(iii) $\Longrightarrow$ (iv). A canonical base is in the definable closure of a Morley sequence.

(iv) $\Longrightarrow$ (i). We have $\hat{a} \in \operatorname{dcl}(A), a^{c} \in \operatorname{bdd}(P(A))$ and $a \downarrow_{a^{c}} P(A)$, so we may consider $(A, P)$ as a free extension of $\left(\hat{a}, a^{c}\right)$. The same holds for $\left(\hat{b}, b^{c}\right) \subseteq$ $(B, P)$, and now apply amalgamation.

$\mathrm{QED}_{1.8}$

We need a tool that would tell us when two types belong to the same Morley class, and this tool is the notion of concurrently indiscernible sequences. In fact, we prove something a bit stronger than we actually need:

Definition 1.9. We say that sequences $\left\{\left(a_{i}^{j}: j<\alpha\right): i<\beta\right\}$ are concurrently indiscernible over $b$ if for every $i<\beta$ and $j_{0}<\alpha$ the sequence $\left(a_{i}^{j}: j_{0} \leq j<\alpha\right)$ is indiscernible over $b \cup\left\{a_{i^{\prime}}^{j}: j<j_{0}, i^{\prime}<\beta\right\}$ (in other word, if every tail is indiscernible over the union of all corresponding heads).

Notation 1.10. Let $\mathrm{S}_{\alpha}^{\text {ind }}(T) \subseteq \mathrm{S}_{\alpha \times \omega}(T)$ denote the set of types of indiscernible sequences of $\alpha$-tuples. In particular, $\operatorname{mcl}(a) \subseteq \mathrm{S}_{|a|}^{\text {ind }}(T)$. 
Lemma 1.11. Assume $A=\left\{a_{i}: i<\beta\right\}$ are tuples, not necessarily disjoint, in some (e.c.) model of $T$, and $q_{i} \in \mathrm{S}_{\left|a_{i}\right|}^{\text {ind }}(T)$ for every $i<\beta$.

Then the following are equivalent:

(i) There is some pair $(D, P)$ where $D \supseteq A$ and $q_{i} \in \operatorname{mcl}^{(D, P)}\left(a_{i}\right)$ for every $i$.

(ii) There are concurrently indiscernible sequences $\left(b_{i}^{j}: j \leq \omega\right)$ with $b_{i}^{\omega}=a_{i}$ and $b_{i}^{<\omega} \vDash q_{i}$ for every $i$.

Proof. $\quad($ i $) \Longrightarrow$ (ii). For every $i<\beta$, find a Morley sequence $\left(b_{i}^{j}: j \leq \omega\right)$ over $P$ such that $b_{i}^{\omega}=a_{i}$ and $b_{i}^{<\omega} \vDash q_{i}$. Write $B^{k}=\left\{b_{i}^{k}: i<\beta\right\}$.

We now give a construction by induction on $k<\omega$. At the beginning of the $k$ th step we assume that $\left(b_{i}^{j}: k \leq j \leq \omega\right)$ is a Morley sequence over $P B^{<k}$ for every $i$. During the step we may move $\left(b_{i}^{j}: k \leq j<\omega\right)$ around a bit in order to obtain the same thing for $k+1$ without moving $A B^{<k}$, nor changing $\operatorname{tp}\left(b_{i}^{<\omega} / a_{i} P B^{<k}\right)$. From this point onward, $B^{k}$ is fixed as well.

We may assume for every $i$ that $b_{i}^{<\omega} \downarrow_{a_{i} P B<k} A$, whereby $b_{i}^{<\omega} \downarrow_{P B<k} A$. We may further assume that $\left\{b_{i}^{<\omega}: i<\beta\right\} \cup\{A\}$ is a $P B^{<k}$-independent set. At this point we fix $B^{k}=\left\{b_{i}^{k}: i<\beta\right\}$ for the rest of the construction, and observe that $B^{k} \downarrow_{P B<k} A$.

We now work for each $i$ separately: we observe that $a_{i} \downarrow_{P B^{<k} b_{i}^{k}} B^{k}$ by the previous paragraph and that $\left(b_{i}^{j}: k<j \leq \omega\right)$ is a Morley sequence over $P B^{<k} b_{i}^{k}$ with $a_{i}=b_{i}^{\omega}$. Therefore there is an automorphism fixing $a_{i} P B^{<k} b_{i}^{k}$ that when applied to $\left(b_{i}^{j}: k<j<\omega\right)$ gives an $P B^{\leq k}$-indiscernible sequence, and in fact a Morley sequence over $P B^{\leq k}$, as required. We now fix $\operatorname{tp}\left(b_{i}^{<\omega} / a_{i} P B^{\leq k}\right)$, and the construction continues.

At the end we obtain concurrently indiscernible Morley sequences over $P$ with the required types.

(ii) $\Longrightarrow$ (i). Let $D=A B^{<\omega}$ and $P(D)=D \cap \operatorname{bdd}\left(B^{<\omega}\right)$. Then $(D, P)$ is a pair.

Since $\left(b_{i}^{j}: k \leq j \leq \omega\right)$ is $B^{<k}$-indiscernible, we have $a_{i}=b_{i}^{\omega} \downarrow_{b_{i}^{[k, \omega)}} B^{<k}$ for every $k<\omega$, whereby $a_{i} \downarrow_{b_{i}^{<\omega}} P(D)$, and $a_{i}^{c}=\mathrm{Cb}\left(b_{i}^{\omega} / b_{i}^{<\omega}\right)$.

Since $\left(b_{i}^{j}: j \leq \omega\right)$ is an indiscernible sequence it is a Morley sequence over $a_{i}^{c}$, and $\operatorname{tp}\left(b_{i}^{<\omega}\right) \in \operatorname{mcl}^{(D, P)}\left(a_{i}\right)$.

$\mathrm{QED}_{1.11}$

Notation 1.12. (i) For $p \in \mathrm{S}(\mathfrak{P})$ define $\operatorname{mcl}(p)$ as $\operatorname{mcl}(a)$ for any $a \vDash p$ : by Lemma 1.8 this is well defined. Similarly, for a set $F \subseteq \mathrm{S}_{\alpha}(\mathfrak{P})$, we define $\operatorname{mcl}(F)=\bigcup_{p \in F} \operatorname{mcl}(p)=\bigcup_{\operatorname{tp}^{\mathfrak{F}}(a) \in F} \operatorname{mcl}(a)$.

(ii) For tuples $a_{<\omega}$ and $b_{<\omega}$ (in an e.c. model of $T$ ) such that all $a_{i}$ and $b_{i}$ are of the same length $\alpha$, say that $a_{<\omega}={ }^{\mathrm{mcl}} b_{<\omega}$ if there exist $a_{\omega}=b_{\omega}$ such that $\left(a_{i}: i \leq \omega\right)$ and $\left(b_{i}: i \leq \omega\right)$ are concurrently indiscernible. Since $T$ is thick, this property is defined by a partial type $r_{\alpha}\left(x_{<\omega}, y_{<\omega}\right)$. We usually omit the subscript $\alpha$ since it can be deduced from the context.

Then Lemma 1.11 gives:

Corollary 1.13. (i) If $q, q^{\prime} \in \mathrm{S}_{\alpha \times \omega}(T)$, then $x_{<\omega}={ }^{\mathrm{mcl}} y_{<\omega} \wedge q\left(x_{<\omega}\right) \wedge q^{\prime}\left(y_{<\omega}\right)$ is consistent if and only if there is $p \in \operatorname{tp}_{\alpha}(\mathfrak{P})$ such that $q, q^{\prime} \in \operatorname{mcl}(p)$. 
(ii) Let $p \in \mathrm{S}_{\alpha}(\mathfrak{P})$ and $q \in \operatorname{mcl}(p)$. Then the partial type $\exists y_{<\omega}\left[r\left(y_{<\omega}, x_{<\omega}\right) \wedge\right.$ $\left.q\left(y_{<\omega}\right)\right]$ defines the set $\operatorname{mcl}(p)$, which is in particular closed. (An existential quantification on a partial type is equivalent to a partial type, by compactness.)

In particular, we may identify $\operatorname{mcl}(p)$ with the partial type $\exists y_{<\omega}\left[r\left(x_{<\omega}, y_{<\omega}\right) \wedge q\left(y_{<\omega}\right)\right]$ for any $q \in \operatorname{mcl}(p)$.

In addition if $a_{i}$ and $b_{i}$ are $\alpha$-tuples for $i<\omega$, then $a_{<\omega}={ }^{\text {mcl }} b_{<\omega}$ if and only if $a_{<\omega}^{\prime}={ }^{\mathrm{mcl}} b_{<\omega}^{\prime}$ for every possible choice of corresponding sub-tuples $a_{i}^{\prime} \subseteq a_{i}, b_{i}^{\prime} \subseteq b_{i}$. It follows that $\mathfrak{P}$-types satisfy the local character, namely the types of two infinite tuples are equal if and only if the types of every two corresponding finite sub-tuples are equal. We conclude that $\mathrm{S}(\mathfrak{P})$ is a set type-space functor.

It is time now to define a language for $\mathfrak{P}$ :

Definition 1.14. Let $\varphi\left(x_{<k}\right) \in \Delta$, where each $x_{i}$ is an $n$-tuple. We define $R_{\varphi}$ as the set of all $p \in \mathrm{S}_{n}(\mathfrak{P})$ such that there is $q\left(x_{<\omega}\right) \in \operatorname{mcl}(p)$ satisfying $\varphi\left(x_{<k}\right)$ (that is to say that $\operatorname{mcl}(p)$ is consistent with $\varphi$ ).

We interpret $R_{\varphi}$ as an $n$-ary predicate on pairs in the obvious way: if $(A, P) \in \mathfrak{P}$ and $a \in A^{n}$ then $(A, P) \vDash R_{\varphi}(a) \Longleftrightarrow \operatorname{tp}^{(A, P)}(a) \in R_{\varphi}$.

We define $\mathcal{L}^{\mathfrak{P}}$ as the set of all such predicates, so $\left|\mathcal{L}^{\mathfrak{P}}\right|=|\mathcal{L}|$. We also define $\Delta^{\mathfrak{P}}=$ $\Delta_{0}\left(\mathcal{L}^{\mathfrak{P}}\right)$, that is the positive quantifier-free formulas in $\mathcal{L}^{\mathfrak{P}}$.

Remark 1.15. We cheat a bit, since $R_{\varphi}$ depends not only on $\varphi$ but on the actual decomposition of its free variables into $k n$-tuples, but we are just going to consider that this information is contained in $\varphi$.

Ordinarily, the set of quantifier-free formulas is closed under conjunction, disjunction and change of variables. We recall that if $f: n \rightarrow m$ is a map and $\varphi\left(x_{<n}\right)$ a formula, then $\psi\left(y_{<m}\right)=\varphi\left(y_{f(0)}, \ldots, y_{f(n-1)}\right)$ is obtained from $\varphi$ through a change of variables by $f$, and we may also write $\psi=f_{*}(\varphi)$. However, in this particular language, the finite disjunction and change of variables are not necessary:

Lemma 1.16. (i) Let $R_{\varphi}\left(x^{<n}\right)$ be an $n$-ary predicate in this language, where $\varphi\left(x_{0}^{<n}, \ldots, x_{k-1}^{<n}\right) \in \Delta$. Let $y^{<m}$ be another tuple of variables and $f: n \rightarrow m$ a map, and let us convene that by $y^{f(<n)}$ we mean the tuple $y^{f(0)}, \ldots, y^{f(n-1)}$. Then the formula $f_{*}\left(R_{\varphi}\right)\left(y^{<m}\right)=R_{\varphi}\left(y^{f(<n)}\right)$ is equivalent to $R_{\psi}\left(y^{<m}\right)$ where $\psi\left(y_{0}^{<m}, \ldots, y_{k-1}^{<m}\right)=\varphi\left(y_{0}^{f(<n)}, \ldots, y_{k-1}^{f(<n)}\right)$.

(ii) $R_{\varphi} \vee R_{\psi}$ is equivalent to $R_{\varphi \vee \psi}$.

This means that every $n$-ary $\Delta^{\mathfrak{P}}$-formula is equivalent to a conjunction of $R_{\varphi^{-}}$ predicates, as finite disjunctions and changes of variables can be transferred to $\varphi$, and similarly for partial $\Delta^{\mathfrak{P}}$-types.

Lemma 1.17. (i) Let $\rho\left(x_{<\omega}\right)$ be a partial $\Delta$-type, which we may assume to be closed under finite conjunctions, and let $R_{\rho}(x)=\bigwedge_{\varphi\left(x_{<k}\right) \in \rho} R_{\varphi}(x)$. Then $p \vdash$ $R_{\rho}$ if and only if $\operatorname{mcl}(p) \wedge \rho$ is consistent.

(ii) Conversely, if $R_{\varphi}(x) \in \mathcal{L}^{\mathfrak{P}}$, then $\operatorname{mcl}\left(R_{\varphi}\right)$ is defined by the partial type $\exists y_{<\omega}={ }^{\mathrm{mcl}} x_{<\omega} \varphi\left(y_{<k}\right) ;$ and if $\rho(x)=\bigwedge_{i<\lambda} R_{\varphi_{i}}(x)$ then $\operatorname{mcl}(\rho)=$ $\bigwedge_{i<\lambda} \operatorname{mcl}\left(R_{\varphi_{i}}\right)$. 
Proof. (i) Since $\operatorname{mcl}(p)$ is a closed set, we have that $\operatorname{mcl}(p)$ is consistent with $\rho$ if and only if $\operatorname{mcl}(p)$ is finitely consistent with $\rho$ if and only if $p \vdash R_{\rho}$.

(ii) Directly by Corollary 1.13

So let us see now what can be expressed in this language. All the following are easily verifiable:

- Any complete $\mathfrak{P}$-type: for any $p \in \mathrm{S}(\mathfrak{P})$ is defined by $R_{\operatorname{mcl}(p)}$.

- Equality: $x=y$ is defined by $R_{x<\omega=y<\omega}(x, y)$.

- Existential quantification: if $\rho(x, y)$ is a partial $\Delta^{\mathfrak{P}}$-type, and $\operatorname{mcl}(\rho)$ is defined by $\rho^{\prime}\left(x_{<\omega}, y_{<\omega}\right)$, then $\exists y \rho(x, y)$ is defined by $R_{\exists y<\omega} \rho^{\prime}$. Therefore, our assumption that $\Delta$ eliminates the existential quantifier (for $T$ ) implies that so does $\Delta^{\mathfrak{P}}($ for $\mathfrak{P})$.

- Any $\Delta$-formula $\varphi(x)$ : this is just $R_{\varphi\left(x_{0}\right)}$.

- $x \in P$ : take $R_{x_{0}=x_{1}}$.

- Indiscernibility of sequences: write $X=x^{<\omega}$, and let $\rho\left(X_{<\omega}\right)$ say that $\left(x_{<\omega}^{j}\right.$ : $j<\omega$ ) is an indiscernible sequence (which is possible since $T$ is thick). Then $R_{\rho}(X)$ says that $X=\left(x^{j}: j<\omega\right)$ is an indiscernible sequence in the sense of $\mathfrak{P}$. This shows that $\mathfrak{P}$ is thick.

- Equality of types: if $T$ is semi-Hausdorff then $\rho\left(x_{<\omega}, y_{<\omega}\right)=$ $\exists z_{<\omega}\left[x_{<\omega}={ }^{\mathrm{mcl}} z_{<\omega} \equiv y_{<\omega}\right]$ is a partial type, and $R_{\rho}(x, y)$ defines the property $x \equiv y$, so $T^{\mathfrak{P}}$ is semi-Hausdorff as well.

- If inequality is positive in $T$, we may say that $x \notin P$, by $R_{x_{0} \neq x_{1}}$ (this can be improved).

The last thing to prove is that this logic is compact.

Lemma 1.18. Let $\Sigma(X)$ be some partial $\Delta^{\mathfrak{P}}$-type, where $X$ is a possibly infinite tuple. Then $\Sigma$ is realised in $\mathfrak{P}$ if and only if it is finitely realised in $\mathfrak{P}$.

Proof. Write $\operatorname{mcl}(\Sigma)\left(X_{<\omega}\right)=\bigwedge_{x \subseteq X, \varphi(x) \in \Sigma} \operatorname{mcl}(\varphi)\left(x_{<\omega}\right)$. Then $\Sigma$ is realised if and only if $\operatorname{mcl}(\Sigma)$ is consistent if and only if $\operatorname{mcl}(\Sigma)$ is finitely consistent if and only if $\Sigma$ is finitely realised.

$\mathrm{QED}_{1.18}$

And we conclude:

Definition 1.19. $T^{\mathfrak{P}}=\mathrm{Th}_{\Pi \mathfrak{P}}(\mathfrak{P})$ is the negative universal theory of pairs in this language.

Theorem 1.20. $T^{\mathfrak{P}}$ is a thick positive Robinson theory in $\Delta^{\mathfrak{P}}$, and $\mathrm{S}(\mathfrak{P})=\mathrm{S}\left(T^{\mathfrak{P}}\right)$. If $T$ is semi-Hausdorff or Hausdorff, then so is $T^{\mathfrak{P}}$.

If $T$ is complete then so is $T^{\mathfrak{P}}$; otherwise, there is a bijection between completions of $T$ and $T^{\mathfrak{P}}$.

Proof. We showed that $\mathcal{L}^{\mathfrak{P}}$ is a language for $\mathfrak{P}$ which can define complete types and satisfies weak compactness. Thus, by [Ben03a], $T^{\mathfrak{P}}$ is a positive Robinson theory in $\Sigma^{\mathfrak{P}}$, and $\mathrm{S}(\mathfrak{P})=\mathrm{S}\left(T^{\mathfrak{P}}\right)$, where $\Sigma^{\mathfrak{P}}$ is the set of positive existential $\mathcal{L}^{\mathfrak{P}}$-formulas. However, as we proved that the language $\Delta^{\mathfrak{P}}$ eliminates the existential quantifier, we can replace $\Sigma^{\mathfrak{P}}$ with $\Delta^{\mathfrak{P}}$.

We also already proved that $T^{\mathfrak{P}}$ is thick, and if $T$ is semi-Hausdorff then so is $T^{\mathfrak{P}}$. 
If $T$ is Hausdorff, and $p \neq p^{\prime} \in \mathrm{S}_{n}\left(T^{\mathfrak{P}}\right)$, then $\operatorname{mcl}(p) \cap \operatorname{mcl}\left(p^{\prime}\right)=\varnothing$, so they can be separated by open sets. In other words, there are partial types $\rho\left(x_{<\omega}\right)$ and $\rho^{\prime}\left(x_{<\omega}\right)$, inconsistent with $\operatorname{mcl}(p)$ and $\operatorname{mcl}\left(p^{\prime}\right)$, respectively, such that $\vDash \rho \vee \rho^{\prime}$. Then $R_{\rho}$ and $R_{\rho^{\prime}}$ are inconsistent with $p$ and $p^{\prime}$, respectively, and $\mathfrak{P} \vDash R_{\rho} \vee R_{\rho^{\prime}}$, so $p$ and $p^{\prime}$ are also separated by open sets.

If $(A, P)$ and $(B, P)$ are two pairs, and $A$ and $B$ embed in e.c. models of the same completion of $T$, then we can amalgamate the two pairs over $(\varnothing, \varnothing)$. On the other hand, if $A$ and $B$ belong to distinct completions of $T$ then we cannot embed them in a single e.c. model. Therefore the completions of $T$ are in bijection with those of $T^{\mathfrak{P}}$.

QED $_{1.20}$

Convention 1.21. We shall work in a universal domain $U^{\mathfrak{P}}$ for (a completion of) $T^{\mathfrak{P}}$.

\section{LOVELY PAIRS}

Since the origin of the theory of pairs is in lovely ones, we need to say something about them.

Definition 2.1. Let $\kappa>|T|$. A pair $(M, P)$ is $\kappa$-lovely if:

(i) For every $A \subseteq M$ such that $|A|<\kappa$, and for every type $p \in S^{T}(A)$, there is $a \vDash p$ in $M$ with $a \downarrow_{A} P(M)$.

(ii) For every $A \subseteq M$ with $|A|<\kappa$ and every type $p \in \mathrm{S}(A)$ which does not divide over $P(A)$, there is $a \vDash p$ in $P(M)$.

Definition 2.2. A set $A$ in $U^{\mathfrak{P}}$ is free if $A \downarrow_{P(A)} P$.

This means that $(A, P)$ is freely embedded in the universal domain, so it determines $\operatorname{tp}^{\mathfrak{P}}(A)$.

Proposition 2.3. Let $\kappa>|T|$. Then a pair $(M, P)$ is a $\kappa$-saturated model of $T^{\mathfrak{P}}$ if and only if it is $\kappa$-lovely.

Proof. Let $(M, P)$ be $\kappa$-saturated, and we want to prove that it is $\kappa$-lovely:

(i) Assume that $A \subseteq M,|A|<\kappa$, and $a$ is some element possibly outside $M$. As we are only interested in $\operatorname{tp}^{T}(a / A)$, we may assume that $a \downarrow_{A} M$.

Set $D=a M, P(D)=D \cap \operatorname{bdd}(P(M))$. Then $(D, P)$ is a pair, and a free extension of $(M, P)$. By saturation, there is an element $a^{\prime} \in M$ such that $\operatorname{tp}^{\mathfrak{P}}(a / A)=\operatorname{tp}^{\mathfrak{P}}\left(a^{\prime} / A\right)$. Define $b=(a A)^{c}$, and $b^{\prime}=\left(a^{\prime} A\right)^{c}$, so:

$$
b, b^{\prime} \in \operatorname{bdd}(P(D))=\operatorname{bdd}(P(M)) \subseteq \operatorname{bdd}(M)
$$

(In fact, since $M$ is $|T|^{+}$-saturated we have $\operatorname{bdd}(M)=\operatorname{dcl}(M)$, but this is not used here.) From $\operatorname{tp}^{\mathfrak{P}}(a / A)=\operatorname{tp}^{\mathfrak{P}}\left(a^{\prime} / A\right)$ we obtain $\operatorname{tp}^{T}(a A b)=\operatorname{tp}^{T}\left(a^{\prime} A b^{\prime}\right)$. Then we have $a^{\prime} A \downarrow_{b^{\prime}} P$, but also:

$$
a \underset{A}{\downarrow} M \Longrightarrow a \underset{A}{\downarrow} b \Longrightarrow a^{\prime} \underset{A}{\downarrow} b^{\prime} \Longrightarrow a^{\prime} \underset{A}{\downarrow} P
$$

as required.

(ii) Assume that $A \subseteq M,|A|<\kappa$, and $a \downarrow_{P(A)} A$. We may assume that $a \downarrow_{P(A)} M$ so $a \downarrow_{P(M)} M$. Let $D=a M$ as above, but define $P(D)=$ 
$D \cap \operatorname{bdd}(a P(M))$. Then $(D, P)$ is a free extension of $(M, P)$, and $\operatorname{tp}^{\mathfrak{P}}(a / A)$ is realised in $M$.

For the converse, assume that $(M, P)$ is $\kappa$-lovely. Assume that $A \subseteq M,|A|<\kappa$ and $a$ is an element of some free extension $(N, P)$ of $(M, P)$. Write $\mu=|A|+|T|<\kappa$. We may find $B \subseteq P(M)$ such that $|B| \leq \mu<\kappa$ and $A \downarrow_{B} P(M)$. Replacing $A$ with $A \cup B$ we may assume that $A$ is free. Now find $C \subseteq P(N)$ such that $|C| \leq \mu<\kappa$ and $a \downarrow_{A C} P(N)$. Since $A$ is free in $M$ it is also free in $N$, so $A \downarrow_{P(A)} C$ and therefore there is $C^{\prime} \subseteq P(M)$ with $C^{\prime} \equiv_{A} C$. Then there is $a^{\prime} \in M$ such that $a^{\prime} C^{\prime} \equiv_{A} a C$ and $a^{\prime} \downarrow_{A C^{\prime}} P(M)$. Then $a C A$ and $a^{\prime} C^{\prime} A$ are both free sets with $a C A \equiv a^{\prime} C^{\prime} A$, whereby $a C A \equiv{ }^{\mathfrak{P}} a^{\prime} C^{\prime} A$, so in particular $a \equiv_{A}^{\mathfrak{P}} a^{\prime}$ as required.

$\mathrm{QED}_{2.3}$

Corollary 2.4. Every pair $(A, P)$ has a free extension to a $\kappa$-lovely pair.

Proof. Just embed it freely in a sufficiently saturated model of $T^{\mathfrak{P}}$.

$\mathrm{QED}_{2.4}$

Remark 2.5. Assume that $T$ is complete, and consider the language $\mathcal{L}_{P}=\mathcal{L} \cup\{P\}$. Then every two $|T|^{+}$-lovely pairs are elementarily equivalent in this language, and any two free sets of cardinality $\leq|T|$ with the same $\mathcal{L}_{P}$-diagram have the same type (this generalises results in [Poi83, BPV03]).

Indeed, since two such sets have the same $\mathfrak{P}$-type, they correspond by an infinite backand-forth in saturated structures. In particular, since the empty set is free, we have the elementary equivalence.

However, this is just a special case of a more general observation: taking any two saturated models of a cat (or in fact, any two equi-universal homogeneous structures), and taking any relational language whose $n$-ary predicates are interpreted as subsets of $\mathrm{S}_{n}$ (without any topological requirement), then they are elementarily equivalent in this language. Of course, they have no reason to be saturated as models of their first-order theory, and when they are not, this first-order theory is rather meaningless.

\section{INDEPENDENCE IN $T^{\mathfrak{P}}$}

3.1. Simplicity. We prove that $T^{\mathfrak{P}}$ is simple and characterise independence.

Proposition 3.1. The following conditions are equivalent for (possibly infinite) tuples $a, b, c$ in $\mathfrak{P}$ :

(i) Whenever $\left(a_{i} b_{i} c_{i}: i<\omega\right) \vDash \operatorname{mcl}(a b c)$, then $b_{<\omega} \downarrow_{a_{<\omega}} c_{<\omega}$.

(ii) There exist $\left(a_{i} b_{i} c_{i}: i<\omega\right) \vDash \operatorname{mcl}(a b c)$ such that $b_{<\omega} \downarrow_{a<\omega} c_{<\omega}$.

(iii) $(a b c)^{c} \in \operatorname{bdd}\left((a b)^{c},(a c)^{c}\right)$ and $\widehat{a b} \downarrow_{\hat{a}} \widehat{a c}$.

(iv) $b \downarrow_{a P} c$ and $\widehat{a b} \downarrow_{\hat{a}} \widehat{a c}$.

(v) $b \downarrow_{a P} c$ and $(a b)^{c} \downarrow_{a^{c}}(a c)^{c}$.

Proof. $\quad($ i) $\Longrightarrow$ (ii). Clear.

(ii) $\Longrightarrow$ (iii). We are given $\left(a_{i} b_{i} c_{i}: i<\omega\right) \vDash \operatorname{mcl}(a b c)$ such that $b_{<\omega} \downarrow_{a_{<\omega}} c_{<\omega}$. This is a Morley sequence in $\operatorname{tp}\left(a b c /(a b c)^{c}\right)$, and we may assume that $a b c=a_{0} b_{0} c_{0}$. In particular, the sequence $\left(a_{i}: 0<i<\omega\right)$ is a Morley sequence over $\hat{a}$, 
indiscernible over $\widehat{a b}$, whereby $\widehat{a b} \downarrow_{\hat{a}} a_{<\omega}$, so:

$$
b_{<\omega} \underset{a<\omega}{\downarrow} c_{<\omega} \Longrightarrow \widehat{a b} \underset{a<\omega}{\downarrow} \widehat{a c} \Longrightarrow \widehat{a b} \underset{\hat{a}}{\downarrow} \widehat{a c}
$$

We also know that $a b \downarrow_{(a b)^{c}} a_{[1, \omega)} b_{[1, \omega)}$, and that $a c \downarrow_{(a c)^{c}}(a b c)^{c} \Longrightarrow$ $a c \downarrow_{(a b)^{c}(a c)^{c}}(a b c)^{c}$. We obtain:

$$
\begin{aligned}
b_{<\omega} \underset{a<\omega}{\downarrow} c_{<\omega} & \Longrightarrow b \underset{a(a b)^{c}}{\downarrow} a_{[1, \omega)} b_{[1, \omega)} c_{<\omega} \Longrightarrow b \underset{a(a b)^{c}}{\downarrow} c(a b c)^{c} \\
& \Longrightarrow a b c \underset{(a b)^{c}(a c)^{c}}{\downarrow}(a b c)^{c}
\end{aligned}
$$

Since $\operatorname{tp}\left(a b c /(a b c)^{c}\right)$ does not divide over $(a b)^{c},(a c)^{c}$, we obtain $(a b c)^{c}=$ $\mathrm{Cb}\left(a b c /(a b c)^{c}\right) \in \operatorname{bdd}\left((a b)^{c},(a c)^{c}\right)$.

(iii) $\Longrightarrow($ iv).

$$
\widehat{a b} \underset{\hat{a}}{\downarrow} \widehat{a c} \Longrightarrow b \underset{a(a b)^{c}(a c)^{c}}{\downarrow} c \Longrightarrow b \underset{a(a b c)^{c}}{\downarrow} c
$$

(iv) $\Longrightarrow(\mathrm{v})$.

$$
\widehat{a b} \underset{\hat{a}}{\downarrow} \widehat{a c} \Longrightarrow(a b)^{c} \underset{\hat{a}}{\downarrow}(a c)^{c} \Longrightarrow(a b)^{c} \underset{a^{c}}{\downarrow}(a c)^{c}
$$

$(\mathrm{v}) \Longrightarrow(\mathrm{i})$. We know that $\left(a_{i} b_{i} c_{i}: i<\omega\right)$ is a Morley sequence over $(a b c)^{c}$, so $a_{i} b_{i} c_{i} \downarrow_{(a b c)^{c}} a_{\neq i} b_{\neq i} c_{\neq i}$ for all $i<\omega$. Then:

$$
\begin{aligned}
b \underset{a(a b c)^{c}}{\downarrow} c & \Longrightarrow b \underset{a(a b)^{c}}{\downarrow} c(a b c)^{c} \\
a_{i} b_{i} c_{i} \underset{(a b c)^{c}}{\downarrow} a_{\neq i} b_{\neq i} c_{\neq i} & \Longrightarrow b_{i} \underset{a_{i}(a b)^{c}}{\downarrow} a_{\neq i} b_{<i} c_{<\omega} \Longrightarrow b_{i} \underset{a<\omega(a b)^{c}}{\downarrow} b_{<i} c_{<\omega}
\end{aligned}
$$

By induction on $i$ we obtain $b_{<i} \downarrow_{a_{<\omega}(a b)^{c}} c_{<\omega}$ for all $i$, whereby $b_{<\omega} \downarrow_{a<\omega(a b)^{c}} c_{<\omega}$. Finally, $(a b)^{c} \downarrow_{a^{c}}(a c)^{c}$ gives us:

$$
\begin{aligned}
a_{<\omega} c_{<\omega} \underset{(a c)^{c}}{\downarrow}(a b)^{c} & \Longrightarrow a_{<\omega} c_{<\omega} \underset{a^{c}}{\downarrow}(a b)^{c} \Longrightarrow c_{<\omega} \underset{a_{<\omega}}{\downarrow}(a b)^{c} \\
& \Longrightarrow b_{<\omega} \underset{a<\omega}{\downarrow} c_{<\omega}
\end{aligned}
$$

As required.

$\mathrm{QED}_{3.1}$

Definition 3.2. If any of the equivalent conditions in Proposition 3.1 holds we say that $b \mathfrak{H}_{a}^{\mathfrak{P}} c$.

Remark 3.3. Conditions (iv) and (v) of Proposition 3.1 were proposed independently, in some form or another, by all three authors of [BPV03] as candidates for independence in $T^{\mathfrak{P}}$ (in the case where $T^{\mathfrak{P}}$ is first order).

Theorem 3.4. $T^{\mathfrak{P}}$ is simple, and $b \mathfrak{l}_{a}^{\mathfrak{P}} c$ if and only if $\operatorname{tp}^{\mathfrak{P}}(b / a c)$ does not divide over a. 
Proof. We need to prove that $\mathfrak{f}^{\mathfrak{P}}$ is an independence relation.

By Proposition 3.1, if $a b c \in U^{\mathfrak{P}}$ and $\left(a_{i} b_{i} c_{i}: i<\omega\right) \vDash \operatorname{mcl}(a b c)$, then $\left.b\right\rfloor_{a}^{\mathfrak{B}} c \Longleftrightarrow$ $b_{<\omega} \downarrow_{a_{<\omega}} c_{<\omega}$. This gives immediate proofs for all the properties of an independence relation, with the exception of the independence theorem for Lascar strong types, which we treat separately.

So assume that $\operatorname{lstp}^{\mathfrak{P}}\left(b_{0} / a\right)=\operatorname{lstp}^{\mathfrak{P}}\left(b_{1} / a\right), c_{0} \downarrow_{a}^{\mathfrak{P}} c_{1}$, and $c_{i} \downarrow_{a}^{\mathfrak{P}} b_{i}$ for $i<2$. Then in particular $\widehat{a b}_{0} \equiv_{\hat{a}}^{\text {Ls }} \widehat{a b_{1}}$, and we also have $\widehat{a b}_{i} \downarrow_{\hat{a}} \widehat{a c_{i}}$ for $i<2$ and $\widehat{a c_{0}} \downarrow_{\hat{a}} \widehat{a c_{1}}$. By the independence theorem in $T$ we can find $b, d \downarrow_{\hat{a}}^{\widehat{a c_{0}} \widehat{a c_{1}}}$ such that $b, d \equiv_{\widehat{a c_{i}}} b_{i},\left(a b_{i}\right)^{c}$.

As $\left(a c_{0} c_{1}\right)^{c} \in \operatorname{bdd}\left(\left(a c_{0}\right)^{c}\left(a c_{1}\right)^{c}\right)$ we have in fact $b, d \downarrow_{\hat{a}} \widehat{a c_{0} c_{1}}$, so $d \downarrow_{a^{c}} \widehat{a c_{0} c_{1}} \Longrightarrow$ $d \downarrow_{\left(a c_{0} c_{1}\right)^{c}} a c_{0} c_{1}$. We may therefore realise $d$ in $P$, and then realise $b$ in $U^{\mathfrak{P}}$ such that $b \downarrow_{\widehat{a c_{0} c_{1}, d}} P \Longrightarrow b \downarrow_{a d} c_{0} c_{1} P$. In particular, $a b \downarrow_{d} P$ (since $d \in P$ and $a^{c} \in \operatorname{dcl}(d)$ ), and $d=(a b)^{c}$.

For $i<2$, we get $b \downarrow_{a(a b)^{c}} c_{i} P \Longrightarrow a b c_{i} \downarrow_{(a b)^{c}\left(a c_{i}\right)^{c}} P$. Recall that $\left(a b_{i} c_{i}\right)^{c} \in$ $\operatorname{bdd}\left(\left(a b_{i}\right)^{c}\left(a c_{i}\right)^{c}\right)$, so $a b_{i} c_{i} \downarrow_{\left(a b_{i}\right)^{c}\left(a c_{i}\right)^{c}} P$ as well. This along with $\widehat{a b} \equiv_{\widehat{a c_{i}}} \widehat{a b_{i}}$ yields $b \equiv{ }_{a c_{i}}^{\mathfrak{P}} b_{i}$.

We know that $\widehat{a b} \downarrow_{\hat{a}} \widehat{a c_{0} c_{1}}$, and $b \downarrow_{a(a b)^{c}} c_{0} c_{1} P \Longrightarrow b \downarrow_{a P} c_{0} c_{1}$, so $b \downarrow_{a}^{\mathfrak{P}} c_{0} c_{1}$, as required.

$\mathrm{QED}_{3.4}$

Notice that in the proof of the independence theorem, we used the assumption $\operatorname{lstp}^{\mathfrak{P}}\left(b_{0} / a\right)=\operatorname{lstp}^{\mathfrak{P}}\left(b_{1} / a\right)$ only to conclude that ${\widehat{a b_{0}}}_{\equiv} \equiv_{\hat{a}}^{\mathrm{Ls}} \widehat{a b_{1}}$. This implies that:

Corollary 3.5. For every $a \in U^{\mathfrak{P}}$, bdd $\mathfrak{P}^{\mathfrak{P}}(a)$ is $\mathfrak{P}$-interdefinable with $\operatorname{bdd}(\hat{a})$.

This still holds even if we consider hyperimaginary sorts of $T^{\mathfrak{P}}$ that are not inherited from $T$.

Proof. One inclusion is clear. For the other, let $b_{E} \in \operatorname{bdd}^{\mathfrak{P}}(a)$ be a hyperimaginary. We saw that $\operatorname{tp}^{\mathfrak{P}}(b / \operatorname{bdd}(\hat{a}))$ is an amalgamation base, so it is equivalent to $\operatorname{tp}^{\mathfrak{P}}\left(b / \operatorname{bdd}^{\mathfrak{P}}(a)\right)$ and therefore implies $\operatorname{tp}^{\mathfrak{P}}\left(b / b_{E}\right)$. Then every automorphism of $U^{\mathfrak{P}}$ that fixes $\operatorname{bdd}(\hat{a})$ sends $b$ to another realisation of $\operatorname{tp}^{\mathfrak{P}}\left(b / b_{E}\right)$, and therefore fixes $b_{E}$, so $b_{E} \in \operatorname{dcl}^{\mathfrak{P}}(\operatorname{bdd}(\hat{a}))$.

$\mathrm{QED}_{3.5}$

Corollary 3.6. If $T$ is supersimple, then so is $T^{\mathfrak{P}}$.

Proof. Let $a$ be a singleton and $B=\left\{b^{i}: i<\alpha\right\}$ a set in $U^{\mathfrak{P}}$. Let $\left(a_{j}, B_{j}: j<\omega\right) \vDash$ $\operatorname{mcl}(a, B)$ in $U$, and extend this to a similar $2 \omega$-sequence $\left(a_{j}, B_{j}: j<2 \omega\right)$. By supersimplicity, there are $n<\omega$ and $I \subseteq \alpha$ finite such that $a_{\omega} \downarrow_{a_{<n}, b_{\in[0, n) \cup[\omega, 2 \omega)}^{\in I}} a_{<\omega} B_{<2 \omega}$.

Then for every $m \in[n, \omega)$ we have $a_{\omega} \downarrow_{a_{<m}, b_{[0, m) \cup[\omega, 2 \omega)}^{\in I}} B_{[0, m) \cup[\omega, 2 \omega)}$, and by removing the segment $[m, \omega)$ we obtain $a_{m} \downarrow_{a_{<m, b<\omega} \in I} B_{<\omega}$. On the other hand, increasing $I$ somewhat, though keeping it finite, we may also assume that $a_{<n} \downarrow_{b_{<} \in \omega} B_{<\omega}$. Combined with the previous observations, an easy induction gives $a_{<m} \downarrow_{b<\omega}^{\in I} B_{<\omega}^{<\omega}$ for every $m \in[n, \omega)$, whereby $a_{<\omega} \downarrow_{b \in \omega} B_{<\omega}$.

We conclude that $a \mathfrak{H}_{b \in I}^{\mathfrak{P}} B$, with $|I|<\omega$, as required.

$\mathrm{QED}_{3.6}$

Remark 3.7. The approach we take here for the proof of simplicity and the characterisation of independence in $T^{\mathfrak{P}}$ is completely different than that which appears in 
BPV03. The basic improvement is in the equivalence $b \mathfrak{|}_{a}^{\mathfrak{P}} c \Longleftrightarrow b_{<\omega} \downarrow_{a_{<\omega}} c_{<\omega}$ which does not appear there. Given this equivalence, all that is left to show is the independence theorem, which then gives us at once the simplicity of $T^{\mathfrak{P}}$, the characterisation of dividing, and the characterisation of Lascar strong types.

In fact, not knowing what hyperimaginary sorts in $T^{\mathfrak{P}}$ look like, the only way we know how to prove that $\operatorname{bdd}^{\mathfrak{P}}(a)=\operatorname{dcl}^{\mathfrak{P}}(\operatorname{bdd}(\hat{a}))$ is through the independence theorem, so might just as well obtain the other results at the same time.

Added in proof: Recent results suggest that the "obvious" definition of supersimplicity is too strong for general cats (more precisely, for those where the property $x \neq y$ is not positive). A better (and more permissive) definition appears in Benb] for Hausdorff cats. The analogue Corollary 3.6 for this definition is true, although it does not seem possible to prove it solely with the tools introduced in [Benb].

3.2. Stability. We recall:

Definition 3.8. (i) $T$ is $\lambda$-stable if $\left|\mathrm{S}_{n}(A)\right| \leq \lambda$ for every set $|A| \leq \lambda$.

(ii) $T$ is stable if it is $\lambda$-stable for some $\lambda$.

(iii) $T$ is superstable if it is $\lambda$-stable for every $\lambda \geq 2^{|T|}$.

One can prove along the lines of the classical proof:

Fact 3.9. Let $T$ be any cat.

(i) $T$ is stable if and only if $T$ is $\lambda^{|T|}$-stable for every $\lambda$.

(ii) $T$ is superstable if and only if it is stable and supersimple.

Theorem 3.10. If $T$ is stable or superstable, then so is $T^{\mathfrak{P}}$.

Proof. Assume that $T$ is stable, and count $\mathfrak{P}$-types over a set $A$. Fix a sequence $\left(A_{i}: i<\omega\right) \vDash \operatorname{mcl}(A)$ : for every $a, \operatorname{tp}^{\mathfrak{P}}(a / A)$ is determined by $\operatorname{tp}\left(a_{<\omega} / A_{<\omega}\right)$, for any $a_{<\omega}$ such that $\left(a_{i} A_{i}: i<\omega\right) \vDash \operatorname{mcl}(a A)$ (and such $a_{<\omega}$ always exists). By stability: $\left|S_{\lambda}^{\mathfrak{P}}(A)\right| \leq\left|S_{\lambda+\omega}^{T}\left(A_{<\omega}\right)\right| \leq(|A|+\omega)^{\lambda+|T|}$, so $|A|=\mu^{|T|} \Longrightarrow\left|S_{|T|}^{\mathfrak{P}}(A)\right|=|A|$.

The result of superstable follows from Fact 3.9 and Corollary 3.6 .

$\mathrm{QED}_{3.10}$

3.3. One-basedness. We recall:

Definition 3.11. A simple cat $T$ (not necessarily thick) is one-based if whenever $\left(a_{i}\right.$ : $i<\omega)$ is a Morley sequence in a complete Lascar strong type $p$ then $\operatorname{Cb}(p) \in \operatorname{bdd}\left(a_{i}\right)$ for some (every) $i$.

Lemma 3.12. A cat $T$ is one-based if and only if, whenever $\left(a_{i}: i<\omega\right)$ is an indiscernible sequence, then $\left(a_{i}: 0<i<\omega\right)$ is independent over $a_{0}$.

Proof. Remember that every indiscernible sequence is a Morley sequence over some set $A$ : for example, a copy of the sequence. Setting $c=\operatorname{Cb}\left(a_{i} / A\right),\left(a_{i}\right)$ is a Morley sequence over $c$.

If $T$ is one based, then we have $c \in \operatorname{bdd}\left(a_{0}\right)$, so $a_{i} \downarrow_{c} a_{<i} \Longrightarrow a_{i} \downarrow_{a_{0}} a_{[1, i-1]}$ for every $i$. Conversely, if $\left(a_{i}\right)$ is a Morley sequence in some Lascar strong type $p$ and $c=\mathrm{Cb}(p)$, then $c \in \operatorname{dcl}\left(a_{\geq 2}\right)$ so $a_{1} \downarrow_{c} a_{0} \Longrightarrow c=\mathrm{Cb}\left(a_{1} / c a_{0}\right)$ and $a_{1} \downarrow_{a_{0}} a_{\geq 2} \Longrightarrow a_{1} \downarrow_{a_{0}} c \Longrightarrow$ $c \in \operatorname{bdd}\left(a_{0}\right)$.

$\mathrm{QED}_{3.12}$ 
Proposition 3.13. If $T$ is one-based then so is $T^{\mathfrak{P}}$.

Proof. Let $\left(a^{j}: j<\omega\right)$ be an indiscernible sequence in $U^{\mathfrak{P}}$. Extend $\left(a^{j}: j<\omega\right)$ to a very long $\left(a^{j}: j<\lambda\right)$, and take $\left(a_{i}^{<\lambda}: i<\omega\right) \vDash \operatorname{mcl}\left(a^{<\lambda}\right)$. Considering it rather as a long sequence $\left(a_{<\omega}^{j}: j<\lambda\right)$, we may extract an indiscernible sequence, which shows that there are $\left(a_{i}^{<\omega}: i<\omega\right) \vDash \operatorname{mcl}\left(a^{<\omega}\right)$ such that $\left(a_{<\omega}^{j}: j<\omega\right)$ is indiscernible. Since $T$ is one-based, the sequence $\left(a_{<\omega}^{j}: 0<j<\omega\right)$ is a Morley sequence over $a_{<\omega}^{0}$, whereby $\left(a^{j}: 0<j<\omega\right)$ is a Morley sequence over $a^{0}$.

$\mathrm{QED}_{3.13}$

\section{THE DESCRIPTION OF $T^{\mathfrak{P}}$ IN $T$ AND ITS FUNCTORIALITY}

In the first section we constructed the abstract elementary category $\mathfrak{P}$, defined the language $\Delta^{\mathfrak{P}}$, and proved that $T^{\mathfrak{P}}=\mathrm{Th}_{\Pi_{\mathfrak{P}}}(\mathfrak{P})$ is a positive Robinson theory in $\Delta^{\mathfrak{P}}$, with $\mathrm{S}\left(T^{\mathfrak{P}}\right)=\mathrm{S}(\mathfrak{P})$. In the topology on $\mathrm{S}\left(T^{\mathfrak{P}}\right)$, closed sets are those defined by partial $\Delta^{\mathfrak{P}}$-types, and equipped with this topology it is a compact type-space functor. However, this topology could have been obtained more directly, using the categoric point of view described in [Bena].

Recall that we defined $S_{\alpha}^{\text {ind }}(T)$ as the subset of $S_{\alpha \times \omega}(T)$ which consists of types of indiscernible sequence of $\alpha$-tuples. If $f: \alpha \rightarrow \beta$ is a map, and $f_{\times \omega}: \alpha \times \omega \rightarrow \beta \times \omega$ is its natural extension to $\omega$-tuples, then $f_{\times \omega}^{*}: \mathrm{S}_{\beta \times \omega}(T) \rightarrow \mathrm{S}_{\alpha \times \omega}(T)$ restricts to a map $f^{\text {ind }}: \mathrm{S}_{\beta}^{\text {ind }}(T) \rightarrow \mathrm{S}_{\alpha}^{\text {ind }}(T)$, so $\mathrm{S}^{\text {ind }}(T)$ is a sub-functor of $\mathrm{S}_{\times \omega}(T)$.

Lemma 4.1. (i) For every $\alpha$ there is a unique map $\mathfrak{d}_{\alpha}: \mathrm{S}_{\alpha}^{\text {ind }}(T) \rightarrow \mathrm{S}_{\alpha}\left(T^{\mathfrak{P}}\right)$ satisfying $\mathfrak{d}_{\alpha}(q)=p \Longleftrightarrow q \in \operatorname{mcl}(p)$.

(ii) For closed sets $F \subseteq \mathrm{S}_{\alpha}^{\text {ind }}(T)$ and $F^{\prime} \subseteq \mathrm{S}_{\alpha}\left(T^{\mathfrak{P}}\right)$, we have $\mathfrak{d}_{\alpha}(F)=R_{F}$ and $\mathfrak{d}_{\alpha}^{-1}\left(F^{\prime}\right)=\operatorname{mcl}\left(F^{\prime}\right)$, and these sets are closed. In particular, every $\mathfrak{d}_{\alpha}$ is continuous and closed.

(iii) Let $f: \alpha \rightarrow \beta$ be a map. Then the following diagram commutes, which makes $\mathfrak{d}: \mathrm{S}^{\text {ind }}(T) \rightarrow \mathrm{S}\left(T^{\mathfrak{P}}\right)$ a morphism of functors:

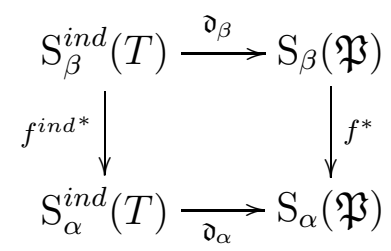

Moreover, if $p \in \mathrm{S}_{\alpha}\left(T^{\mathfrak{P}}\right), q \in \mathfrak{d}_{\alpha}^{-1}(p)=\operatorname{mcl}(p) \subseteq \mathrm{S}_{\alpha}^{\text {ind }}(T)$ and $p^{\prime} \in f^{*-1}(p)=$ $f_{*}(p) \subseteq \mathrm{S}_{\beta}\left(T^{\mathfrak{P}}\right)$, then there is $q^{\prime} \in \operatorname{mcl}\left(p^{\prime}\right) \cap f_{*}^{\text {ind }}(\operatorname{mcl}(p)) \subseteq \mathrm{S}_{\beta}^{\text {ind }}(T)$.

(iv) $\mathfrak{d}: \mathrm{S}^{\text {ind }}(T) \rightarrow \mathrm{S}\left(T^{\mathfrak{P}}\right)$ is a quotient map, meaning that $\mathfrak{d}$ is a surjective map, and the topology on $\mathrm{S}\left(T^{\mathfrak{P}}\right)$ is maximal such that $\mathfrak{d}$ is continuous.

Proof. (i) For every $q \in \mathrm{S}_{\alpha}^{\text {ind }}(T)$ there is at most one value that $\mathfrak{d}_{\alpha}$ can take, since $p \neq p^{\prime} \Longrightarrow \operatorname{mcl}(p) \cap \operatorname{mcl}\left(p^{\prime}\right)=\varnothing$. Such a value always exists, as can be seen by applying Lemma 1.11 with $\beta=1$.

(ii) This is just what Lemma 1.17 says.

(iii) It is a fact that if $a, b$ and $P$ are given, then a sequence $\left(a_{i}: i<\omega\right)$ is a Morley sequence in $\operatorname{tp}(a / P)$ if and only if there are $b_{<\omega}$ such that $\left(a_{i} b_{i}: i<\omega\right)$ is a 
Morley sequence in $\operatorname{tp}(a b / P)$. Then commutativity is one direction, and the moreover part is the other.

(iv) Each $\mathfrak{d}_{\alpha}$ is surjective since $\operatorname{mcl}(p) \neq \varnothing$ for every $p \in \mathrm{S}_{\alpha}(\mathfrak{P})$. A surjective, closed and continuous map is a quotient map.

$\mathrm{QED}_{4.1}$

Thus, we could have defined the topology on $\mathrm{S}(\mathfrak{P})$ from the beginning as the quotient topology, without ever bothering to define a language explicitly. Then, the commutativity statement in Lemma 4.1.(iii)] shows that $f^{*}: \mathrm{S}_{\beta}(\mathfrak{P}) \rightarrow \mathrm{S}_{\beta}(\mathfrak{P})$ is continuous, and the moreover part shows that $f^{*}$ is closed. As every set $\operatorname{mcl}(p)$ is closed, the topology on $\mathrm{S}(\mathfrak{P})$ is $T_{1}$, and it is compact as the quotient of a compact topology.

In short, we could have skipped everything that comes after Corollary 1.13, and still conclude that $\mathrm{S}(\mathfrak{P})$ is a compact type-space functor, so there is a positive Robinson theory $T^{\mathfrak{P}}$ in some language such that $\mathrm{S}(\mathfrak{P})=\mathrm{S}\left(T^{\mathfrak{P}}\right)$, but this time also as topological functors. In fact, we could have skipped the entire first section, constructing $\mathrm{S}(\mathfrak{P})$ directly as the quotient of $S^{i n d}(T)$ by the appropriate equivalence relation (but then, of course, we wouldn't know what it is that we are constructing).

This very abstract approach still seems (at least to the author) quite convenient, and allows a few elegant observations. Recall from Bena:

Definition 4.2. $\quad$ (i) Let $\alpha_{\mathfrak{d}}$ be an ordinal and $S, S^{\prime}$ compact type-space functors. Let $\mathfrak{d}: S_{\times \alpha_{\mathfrak{d}}} \rightarrow S^{\prime}$ be a continuous partial map, meaning that $\left(S_{\times \alpha_{\mathfrak{d}}}\right)_{I}=S_{I \times \alpha}$, $\operatorname{dom}(\mathfrak{d}) \subseteq S_{\times \alpha_{\mathfrak{d}}}$ is a closed sub-functor, and $\mathfrak{d}: \operatorname{dom}(\mathfrak{d}) \rightarrow S^{\prime}$ is a continuous surjective morphism of functors. If $\varphi\left(x_{<n}\right)$ is a formula in the language of $S^{\prime}$ identify it with the closed set it defines $\varphi \subseteq S_{n}^{\prime}$, and let $\tilde{\varphi}\left(\bar{x}_{<n}\right)$ be the partial type in the language of $S$ defining $\mathfrak{d}_{n}^{-1}(\varphi) \subseteq S_{n \times \alpha_{0}}$.

Let $x_{<n+m}$ be a tuple of variables, and let:

$$
\begin{aligned}
& \psi\left(x_{<n}\right)=\exists x_{\in[n, n+m)} \bigwedge_{j<l} \varphi_{j}\left(x_{i_{j, 0}}, \ldots, x_{i_{j, k_{j}-1}}\right) \\
& \hat{\psi}\left(\bar{x}_{<n}\right)=\exists \bar{x}_{\in[n, n+m)} \bigwedge_{j<l} \tilde{\varphi}_{j}\left(\bar{x}_{i_{j, 0}}, \ldots, \bar{x}_{i_{j, k_{j}-1}}\right),
\end{aligned}
$$

where each $\varphi_{j}$ is an $k_{j}$-ary formula, and $i_{j, s}<m+n$ for $j<l$ and $s<k_{j}$.

Then $\left(\mathfrak{d}, \alpha_{\mathfrak{d}}\right)$ is a description of $S^{\prime}$ in $S$, written $\mathfrak{d}: S \rightarrow S^{\prime}$, if whenever $\psi, \hat{\psi}$ are as above and $p \in \operatorname{dom}(\mathfrak{d})$ is in the right number of variables then:

$$
p \vdash \hat{\psi} \Longleftrightarrow \mathfrak{d}(p) \vdash \psi
$$

(ii) A description is closed if $\mathfrak{d}$ is a closed map.

(iii) If $T$ and $T^{\prime}$ are simple cats, then a morphism $\mathfrak{r}: \mathrm{S}(T) \rightarrow \mathrm{S}\left(T^{\prime}\right)$ preserves independence if whenever $a, b, c$ and $a^{\prime}, b^{\prime}, c^{\prime}$ are possibly infinite tuples in the universal domains of $T$ and $T^{\prime}$, respectively, and $\operatorname{tp}^{T^{\prime}}\left(a^{\prime}, b^{\prime}, c^{\prime}\right)=\mathfrak{r}\left(\operatorname{tp}^{T}(a, b, c)\right)$, then $b \downarrow_{a}^{T} c \Longleftrightarrow b^{\prime} \downarrow_{a^{\prime}}^{T^{\prime}} c^{\prime}$.

Lemma 4.3. Let $q_{j}\left(x_{<k_{j}}^{<\omega}\right)$ be partial types for $j<l$, each of which implying that $\left(x_{<k_{j}}^{s}: s<\omega\right)$ is an indiscernible sequence of $k_{j}$-tuples (in other words, $q_{j} \vdash \operatorname{dom}\left(\mathfrak{d}_{k_{j}}\right)$ ). Assume that $\pi\left(y_{<n}^{<\omega}\right)=\bigwedge_{j<l} q_{j}\left(y_{i_{j, 0}}^{<\omega}, \ldots, y_{i_{j, k_{j}-1}}^{<\omega}\right)$ is consistent, where $i_{j, t}<n$ for every 
$j<l$ and $t<k_{j}$. Then it can be realised in $\operatorname{dom}(\mathfrak{d})$, that is to say that it has a realisation which is an indiscernible sequence of $n$-tuples.

Proof. Fix a very big $\lambda$, and let $\pi^{\prime}\left(y_{<n}^{<\lambda}\right)$ say that $\pi\left(y_{<n}^{<\omega}\right)$, and in addition for every $j<l$ the sequence $\left(y_{i_{j,<k_{j}}}^{s}: s<\lambda\right)$ is indiscernible. Then $\pi^{\prime}$ is consistent by compactness, and let $a_{<n}^{<\lambda} \vDash \pi^{\prime}$. By indiscernibility, we have $a_{<n}^{s<\omega} \vDash \pi$ for every increasing sequence $s_{0}<s_{1}<\cdots<\lambda$. As we took $\lambda$ sufficiently big, we can extract an indiscernible sequence $\left(b_{<n}^{s}: s<\omega\right)$ such that, for every $t<\omega$ there are $s_{0}^{t}<\cdots<s_{t-1}^{t}<\lambda$ such that $b_{<n}^{<t} \equiv a_{<n}^{s_{<t}^{t}}$, whereby $b_{<n}^{<\omega} \vDash \pi$ as required.

$\mathrm{QED}_{4.3}$

Theorem 4.4. $\quad$ (i) The map $\mathfrak{d}: \mathrm{S}^{\text {ind }}(T) \rightarrow \mathrm{S}\left(T^{\mathfrak{P}}\right)$, viewed as a partial map $\mathfrak{d}$ : $\mathrm{S}_{\times \omega}(T) \rightarrow \mathrm{S}\left(T^{\mathfrak{P}}\right)$, is a closed description also noted $\mathfrak{d}: T \rightarrow T^{\mathfrak{P}}$, with a factor $\alpha_{\mathfrak{d}}=\omega$, and domain $\operatorname{dom}(\mathfrak{d})=\mathrm{S}^{\text {ind }}(T)$.

(ii) This description is functorial: if $g: T \rightarrow T^{\prime}$ is any morphism of type-space functors of thick simple cats, then there is a unique morphism $g^{\mathfrak{P}}: T^{\mathfrak{P}} \rightarrow T^{\prime \mathfrak{P}}$ that makes the following diagram commute:

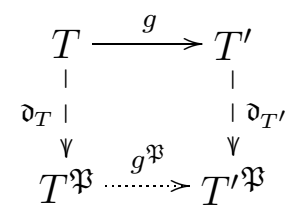

(iii) If $g$ preserves independence, then so does $g^{\mathfrak{P}}$.

Proof. (i) Given Lemma 4.1, all that is left to prove is (1) of Definition 4.2.

$\Longleftarrow$ follows from the moreover part of Lemma 4.1.(iii). $\Longrightarrow$ follows from Lemma 4.3.

(ii) Just verify that if $q, q^{\prime} \in \mathrm{S}_{\alpha}^{\text {ind }}(T)$ belong to the same Morley class, then so do $g(q), g\left(q^{\prime}\right)$.

(iii) This is immediate from Proposition 3.1.

$\mathrm{QED}_{4.4}$

We prove in [Bena] that a theory describable in a simple theory is simple. Thus, had we taken the course proposed in the beginning of this section, we could have concluded that $T^{\mathfrak{P}}$ is simple immediately, even without giving an explicit characterisation of independence.

Recall also from Bena]:

Definition 4.5. Let $T$ be a simple cat and $T^{\prime}$ a stable one. Then a stable representation of $T$ in $T^{\prime}$ is a morphism $\mathfrak{r}: \mathrm{S}(T) \rightarrow \mathrm{S}\left(T^{\prime}\right)$ satisfying the following additional condition (called preservation of independence): If $a, b, c$ are (possibly infinite) tuples in a model of $T, a^{\prime}, b^{\prime}, c^{\prime}$ in a model of $T^{\prime}$ and $\operatorname{tp}^{T^{\prime}}\left(a^{\prime}, b^{\prime}, c^{\prime}\right)=\mathfrak{r}\left(\operatorname{tp}^{T}(a, b, c)\right)$ then $a \downarrow_{b} c \Longleftrightarrow$ $a^{\prime} \downarrow_{b^{\prime}} c^{\prime}$

With a minor abuse of notation we may also write it as $\mathfrak{r}: T \rightarrow T^{\prime}$.

Corollary 4.6. Assume that $T$ is simple and thick and has a thick stable representation (that is $\mathfrak{r}: T \rightarrow T^{\prime}$ where $T^{\prime}$ is stable and thick). Then so does $T^{\mathfrak{P}}$.

Proof. Let $\mathfrak{r}: T \rightarrow T^{\prime}$ be a stable representation. Then $\mathfrak{r}^{\mathfrak{P}}: T^{\mathfrak{P}} \rightarrow T^{\prime \mathfrak{P}}$ preserves independence and $T^{\prime \mathfrak{P}}$ is stable, so it is a stable representation. $\quad \mathrm{QED}_{4.6}$ 
Lastly, we would like to relate the lovely pairs construction with another "standard" construction, namely the addition of a generic automorphism.

Definition 4.7. For stable $T$, we let $\mathcal{C}^{A}(T)$ denote the category of boundedly closed sets from $T$ equipped with an automorphism $\sigma$.

If the category $\mathcal{C}^{A}(T)$ forms a simple cat in a language extending that of $T$, and whose notion of independence is independence in $T$ of $\sigma$-closures, then we denote this cat by $T^{A}$ and say that $T^{A}$ exists.

By $\mathbb{P}$ Pil00], if $T$ is first order then $T^{A}$ exists, and it can be further shown to be Robinson. We do not wish to address here the issue of existence of $T^{A}$ in the general case, so we will just assume that $T^{A}$ exists. We do know however from [Bena] that if $T^{A}$ exists then we have a stable representation $\mathfrak{r}_{A}: T^{A} \rightarrow T_{\times \omega}$, which sends the type of an element in $T^{A}$ to the type in $T$ of its orbit under the automorphism.

Proposition 4.8. Assume that $T^{A}$ does exist as and is thick. Then $\left(T^{\mathfrak{P}}\right)^{A}$ exists and is equal to $\left(T^{A}\right)^{\mathfrak{P}}$, and we have a commutative diagram, where $\mathfrak{r}_{A}$ and $\mathfrak{r}_{A}^{\mathfrak{P}}$ are the stable representations of $T^{A}$ and $T_{A}^{\mathfrak{P}}$, respectively:



Proof. Let $\mathcal{C}^{\mathfrak{P}}\left(T^{A}\right)$ be the category of pairs in $T^{A}$. Let $(A, \sigma) \in \mathcal{C}^{A}\left(T^{\mathfrak{P}}\right)$. Then $A$ is boundedly closed in the sense of $T^{\mathfrak{P}}$, which means that $A^{c} \subseteq \operatorname{dcl}(P(A)$ ) (i.e., $\left.A \downarrow_{P(A)} P\right)$, and both $A$ and $P(A)$ are boundedly closed in the sense of $T$. Writing it as $(A, \sigma, P)$ it can also be viewed as a pair in the sense of $T^{A}$ and therefore an object of $\mathcal{C}^{\mathfrak{P}}\left(T^{A}\right)$.

This mapping from $\mathcal{C}^{A}\left(T^{\mathfrak{P}}\right)$ into $\mathcal{C}^{\mathfrak{P}}\left(T^{A}\right)$ is a full and faithful functor: indeed, if $f:(A, P, \sigma) \rightarrow(B, P, \sigma)$ is a mapping, then it is a morphism in the sense of either category if and only if $f(P(A))=P(f(A)), f \circ \sigma_{A}=\sigma_{B} \circ f$ and $f(A) \downarrow_{f(P(A))} P(B)$ (since $\sigma$ is an automorphism of $A, P(A)$ and $P(B)$, independence in the sense of $T$ and of $T^{A}$ is the same). Moreover, this functor is co-final: every object of $\mathcal{C}^{\mathfrak{P}}\left(T^{A}\right)$ embeds into the image of an object of $\mathcal{C}^{A}\left(T^{\mathfrak{P}}\right)$.

This means that since $\mathcal{C}^{\mathfrak{P}}\left(T^{A}\right)$ is an abstract elementary category so is $\mathcal{C}^{A}\left(T^{\mathfrak{P}}\right)$, and they have the same type-spaces. Therefore these two categories are equivalent for our purposes: we can use the language we chose for $\mathcal{C}^{\mathfrak{P}}\left(T^{A}\right)$ also for $\mathcal{C}^{A}\left(T^{\mathfrak{P}}\right)$, and both have the same positive Robinson theory in this language $\left(T^{A}\right)^{\mathfrak{P}}=\left(T^{\mathfrak{P}}\right)^{A}$. Finally it is an easy exercise to see that independence in the sense of $\left(T^{A}\right)^{\mathfrak{P}}$ coincides with independence in the sense of $T^{\mathfrak{P}}$ of the $\sigma$-closures.

The commutativity of the diagram is also easy.

$\mathrm{QED}_{4.8}$

\section{LOWNESS AND NEGATION}

Definition 5.1. $\quad$ (i) We say that a formula $\varphi$ is clopen if it defines a clopen set in the type-space. Equivalently, if $\neg \varphi$ is equivalent to a positive formula (and then we identify them). 
(ii) We recall that a $k$-inconsistency witness for a formula $\varphi(x, y)$ is a formula $\psi\left(y_{<k}\right)$ such that $\psi\left(y_{<k}\right) \wedge \bigwedge_{i<k} \varphi\left(x, y_{i}\right)$ is inconsistent.

A formula $\varphi(x, y)$ is low if it has a $k$-inconsistency witness $\psi$ such that, for every indiscernible sequence $\left(a_{i}\right),\left\{\varphi\left(x, a_{i}\right)\right\}$ is inconsistent if and only if $\vDash$ $\psi\left(a_{0}, \ldots, a_{k-1}\right)$ (in other words, it has a universal inconsistency witness for indiscernible sequences).

(iii) $T$ is low if every formula is.

We recall that a cat $T$ is Robinson if and only if the type-spaces are totally disconnected if and only if we can choose the language such that all basic formulas are clopen. It is first order if and only if existential formulas are clopen as well.

Remark 5.2. If $T$ is first order then $\varphi(x, y)$ is low if and only if there is $k<\omega$ such that, if $\left(a_{i}: i<\omega\right)$ is indiscernible and $\left\{\varphi\left(x, a_{i}\right)\right\}$ is inconsistent then it is $k$-inconsistent. The proofs of several of the results below can be simplified accordingly. However, note that being Robinson does not suffice, since we need the negation of $\exists x \bigwedge_{i<k} \varphi\left(x, y_{i}\right)$, and this is an existential formula.

Notation 5.3. Let $\varphi(x, y)$ be a $T$-formula. Then $\varphi(P, y)=\exists x \in P \varphi(x, y)$ is positive. If $\psi$ is a $k$-inconsistency witness for $\varphi$ then $\neg_{\psi} \varphi(P, x)=R_{\psi}(y)$ is positive as well.

If the existential formula $\psi(\bar{y})=\exists x \bigwedge_{i<k} \varphi\left(x, y_{i}\right)$ defines a clopen set, then we write $\neg_{k} \varphi(P, y)=R_{\neg \psi}(y)$.

Lemma 5.4. If $A \subseteq B$ and $a \downarrow_{A} B$ then $\varphi(x, a)$ divides over $A$ if and only if it divides over $B$.

Proof. A Morley sequence for $a$ over $B$ is also a Morley sequence over $A$. $\quad$ QED $_{5.4}$

Lemma 5.5. If $a$ and $b$ are tuples in $U^{\mathfrak{P}}$ satisfying precisely the same $\varphi(P, y)$ formulas, then $a \equiv{ }^{\mathfrak{P}} b$.

Proof. Let $A \subseteq P$ be such that $a \downarrow_{A} P$. Since $b$ satisfied all $\varphi(x, P)$ predicates that $a$ does, then by compactness, we can find $B \subseteq P$ such that $a A \equiv b B$. Assume now that $\vDash \varphi(c, b)$ for some $c \in P$. Then $\vDash \varphi(P, b) \Longrightarrow \vDash \varphi(P, a)$, whereby $\varphi(x, a)$ does not divide over $P$ and therefore neither over $A$ (since $a \downarrow_{A} P$ ). Then $\varphi(x, b)$ does not divide over $B$ either and $b \downarrow_{B} P$. This suffices to see that $a \equiv \mathfrak{P} b . \quad$ QED $_{5.5}$

Lemma 5.6. Let a be a tuple in $U^{\mathfrak{P}}$ and $\varphi(x, y)$ a $T$-formula. Then $\varphi(x, a)$ does not divide over $P$ if and only if it is satisfied in $P$.

Proof. If $\varphi(x, a)$ is realised in $P$, clearly it cannot divide over $P$. Conversely, assume that it does not divide over $P$. Then there is a complete type $p \in \mathrm{S}(a P)$ such that $p(x) \vdash \varphi(x, a)$ and $p$ does not divide over $P$. By loveliness of the universal domain, we can realise $p$ in $P$.

$\mathrm{QED}_{5.6}$

Corollary 5.7. $\mathfrak{P} \vDash \neg \varphi(P, y) \leftrightarrow \bigvee_{\psi} \neg_{\psi} \varphi(P, y)$, where $\psi$ varies over all inconsistency witnesses for $\varphi$.

Proof. $\varphi(x, a)$ is not satisfied in $P$ if and only if it divides over $P$ if and only if there is a Morley sequence $\left(a_{i}: i<\omega\right)$ for $a$ over $P$ such that $\left\{\varphi\left(x, a_{i}\right)\right\}$ is inconsistent if and only if there is $\left(a_{i}: i<\omega\right) \vDash \operatorname{mcl}(a)$ satisfying an inconsistency witness for $\varphi$. QED $_{5.7}$ 
Corollary 5.8. If $a$ and $b$ are tuples in $\mathfrak{P}$, and $b$ satisfies every formula of the form $\varphi(P, y)$ or $\neg_{\psi} \varphi(P, y)$ that a does, then $a \equiv \mathfrak{P} b$.

Proof. In this case $a$ and $b$ satisfy precisely the same $\varphi(P, y)$ formulas.

$\mathrm{QED}_{5.8}$

Lemma 5.9. A formula $\varphi(x, y)$ is low if and only if for every $\lambda$ there is a partial type $\Phi_{\varphi}^{\mathrm{div}}(y, Z),|Z|=\lambda$, such that $\varphi(x, a)$ divides over a set $B$ of cardinality $\lambda$ if and only if $\vDash \Phi_{\varphi}^{\operatorname{div}}(a, B)$.

Proof. Assume that $\varphi$ is low, and let $\psi$ be the universal inconsistency witness. Then the partial type saying that there is a $Z$-indiscernible sequence $\left(y_{i}: i<\omega\right)$ satisfying $y_{0}=y \wedge \psi\left(y_{0}, \ldots, y_{k-1}\right)$ will do.

For the converse, write the partial type saying that $\left(y_{i}: i \leq \omega\right)$ is indiscernible, $\exists x \bigwedge_{i<k} \varphi\left(x, y_{i}\right)$ for every $k<\omega$, and $\varphi\left(x, y_{\omega}\right)$ divides over $y_{<\omega}$. If this could be realised, we could continue the sequence to length $2 \omega$, in which case $\left(y_{i}: \omega \leq i<2 \omega\right)$ would be a Morley sequence over $y_{<\omega}$, whereby $\varphi\left(x, y_{\omega}\right)$ cannot divide over $y_{<\omega}$. Then this is inconsistent, so there are $k_{0}<\omega, \psi_{0}$ implied by the statement that the sequence is indiscernible, and $\psi_{1}(\bar{y}) \in \Phi_{\varphi}^{\mathrm{div}}\left(y_{\omega}, y_{<\omega}\right)$, such that $\psi_{0}(\bar{y}) \wedge \psi_{1}(\bar{y}) \wedge \exists x \bigwedge_{i<k_{0}} \varphi\left(x, y_{i}\right)$ is contradictory. Let $k$ be the total number of $y_{i}$ appearing there, and write $\psi_{0} \wedge$ $\psi_{1}=\psi\left(y_{0}, \ldots, y_{k-1}\right)$. Then $\psi$ is a $k$-inconsistency witness for $\varphi$, and we claim that it is universal. Indeed, assume that $\left(a_{i}: i<\omega\right)$ are indiscernible and $\left\{\varphi\left(x, a_{i}\right)\right\}$ is inconsistent. Let $a_{\omega}$ continue this sequence, so $\varphi\left(x, a_{\omega}\right)$ divides over $a_{<\omega}$ : then $\psi_{0}$ holds due to the indiscernibility, and $\psi_{1}$ since $\vDash \Phi_{\varphi}^{\text {div }}\left(a_{\omega}, a_{<\omega}\right)$. This shows that $\psi$ witnesses that $\varphi$ is low.

$\mathrm{QED}_{5.9}$

Remark 5.10. The converse part was first proved in a special case by Vassiliev.

Corollary 5.11. If $\varphi$ is low, then $\varphi(P, y)$ is clopen in $T^{\mathfrak{P}}$. The converse holds if $T$ is Robinson.

Proof. For left to right, if $\psi(\bar{y})$ witnesses that $\varphi(x, y)$ is low, then $\mathfrak{P} \vDash \neg \psi \varphi(P, y) \leftrightarrow$ $\neg \varphi(P, y)$.

For the converse, assume that $T$ is Robinson, and that all the formulas are clopen. Set $\Phi_{\varphi}^{\text {div }}(y, Z)=\bigcap\{\operatorname{tp}(a, B): \varphi(x, a)$ divides over $B\}$, and it will be enough to show that $\vDash \Phi_{\varphi}^{\text {div }}(a, B) \Longrightarrow \varphi(x, a)$ divides over $B$.

Assume then that $\vDash \Phi_{\varphi}^{\operatorname{div}}(a, B)$, and set $q(y, Z)=\operatorname{tp}(a, B)$. For every formula $\chi$ we have $\chi \in q$ if and only if $\neg \chi \notin q$ if and only if there are $a^{\prime}, B^{\prime}$ such that $\vDash \chi\left(a^{\prime}, B^{\prime}\right)$ and $\varphi\left(x, a^{\prime}\right)$ divides over $B^{\prime}$. We can realise $B^{\prime}$ in $P$ and then realise $a^{\prime}$ such that $a^{\prime} \downarrow_{B^{\prime}} P$. Then $\varphi\left(x, a^{\prime}\right)$ divides over $P$, and $\vDash \neg \varphi\left(P, a^{\prime}\right)$.

This shows that $\neg \varphi(P, y) \wedge Z \subseteq P \wedge q(y, Z)$ is finitely consistent. As $\neg \varphi(P, y)$ is positive, this is consistent, and we might just as well assume that it is realised by $a, B$. But then $\neg \varphi(P, a) \Longrightarrow \varphi(x, a)$ divides over $B$.

QED $_{5.11}$

Corollary 5.12. $T$ is low if and only if every formula $\varphi(P, y)$ is clopen. In this case $T$ is first-order, and the formulas $\varphi(P, y), \neg \varphi(P, y)$ form a basis for the $\mathcal{L}^{\mathfrak{P}}$, so taking them as basic formulas $T^{\mathfrak{P}}$ is Robinson.

Proof. If $T$ is low then we know that every formula $\varphi(P, y)$ is clopen. Conversely, we know that $P$ is a model of $T$, so if every formula $\varphi(P, y)$ is clopen then $T$ is first order (existential formulas are clopen), and then we know that $T$ is low.

QED $_{5.12}$ 
Remark 5.13. If fact, when $T$ is low with quantifier elimination, we can axiomatise $T^{\mathfrak{P}}$ directly as a universal Robinson theory in the language $\mathcal{L}^{\prime}$ consisting of predicates $\varphi(P, y)$ for every formula $\varphi(x, y)$ in the language of $T$ :

For every $n, m<\omega$ and formulas $\varphi_{i}\left(x_{i}, y_{i}\right)$ for $i<n$ and $\psi_{j}\left(t_{j}, z_{j}\right)$ for $j<m$, consider the statement:

$$
\forall y_{<n} z_{<m}\left[\bigwedge_{i<n} \varphi_{i}\left(P, y_{i}\right) \wedge \bigwedge_{j<m} \neg \psi_{j}\left(P, z_{j}\right)\right] \rightarrow \exists x_{<n} \bigwedge_{i<n} \varphi_{i}\left(x_{i}, y_{i}\right) \wedge \bigwedge_{j<m} \Phi_{\psi_{j}}^{\mathrm{div}}\left(z_{i}, x_{<n}\right)
$$

Since $T$ is assumed to have quantifier elimination, the statement $\exists x_{<n} \bigwedge_{i<n} \varphi_{i}\left(x_{i}, y_{i}\right) \wedge$ $\bigwedge_{j<m} \Phi_{\psi_{j}}^{\mathrm{div}}\left(z_{i}, x_{<n}\right)$ is equivalent modulo $T$ to a quantifier-free partial type. Therefore, the statement above can be viewed as a universal theory in $\mathcal{L}^{\prime}$. Take $T^{\prime}$ to be the universal theory consisting of all universal $\mathcal{L}^{\prime}$-sentences thus obtained. Then $T^{\prime}$ is a Robinson theory, equivalent as a cat to $T^{\mathfrak{P}}$ (that is, has the same type-space).

This is proved in BPV03].

We know that a stable theory is low if and only if it is first-order: one direction is classical, the other was proved above. We can also prove:

Proposition 5.14. If $T$ is stable and Robinson then so is $T^{\mathfrak{P}}$.

Proof. Since $T$ is stable, $\operatorname{mcl}(p)$ is a complete type for every $p$, whereby $\neg R_{\varphi}=R_{\neg \varphi}$ for every formula $\varphi$, and $T^{\mathfrak{P}}$ is Robinson.

$\mathrm{QED}_{5.14}$

Question 5.15. Find a necessary and sufficient condition for $T^{\mathfrak{P}}$ to be Robinson.

\section{REFERENCES}

[Bena] Itay Ben-Yaacov, Thickness, and a categoric view of type-space functors, Fundamenta Mathematicae (to appear).

[Benb] - Uncountable dense categoricity in cats, preprint.

[Ben03a] , Positive model theory and compact abstract theories, Journal of Mathematical Logic 3 (2003), no. 1, 85-118.

[Ben03b] _ Simplicity in compact abstract theories, Journal of Mathematical Logic 3 (2003), no. 2, 163-191.

[BPV03] Itay Ben-Yaacov, Anand Pillay, and Evgueni Vassiliev, Lovely pairs of models, Annals of Pure and Aplied Logic 122 (2003), 235-261.

[HKP00] Bradd Hart, Byunghan Kim, and Anand Pillay, Coordinatisation and canonical bases in simple theories, Journal of Symbolic Logic 65 (2000), 293-309.

[Kim98] Byunghan Kim, Forking in simple unstable theories, Journal of the London Mathematical Society 57 (1998), no. 2, 257-267.

[KP97] Byunghan Kim and Anand Pillay, Simple theories, Annals of Pure and Applied Logic 88 (1997), 149-164.

[Pil00] Anand Pillay, Forking in the category of existentially closed structures, Connections between Model Theory and Algebraic and Analytic Geometry (Angus Macintyre, ed.), Quaderni di Matematica, vol. 6, University of Naples, 2000.

[Poi83] Bruno Poizat, Paires de structures stables, Journal of Symbolic Logic 48 (1983), no. 2, 239-249.

Itay Ben-Yaacov, Massachusetts Institute of Technology, Department of Mathematics, 77 Massachusetts Avenue, Room 2-101, Cambridge, MA 02139-4307, USA

E-mail address: pezz@math.mit.edu

$U R L:$ http: //www-math.mit.edu/ pezz 